\title{
A mathematical formulation and an NSGA-II algorithm for minimizing the makespan and energy cost under time-of-use electricity price in an unrelated parallel machine scheduling
}

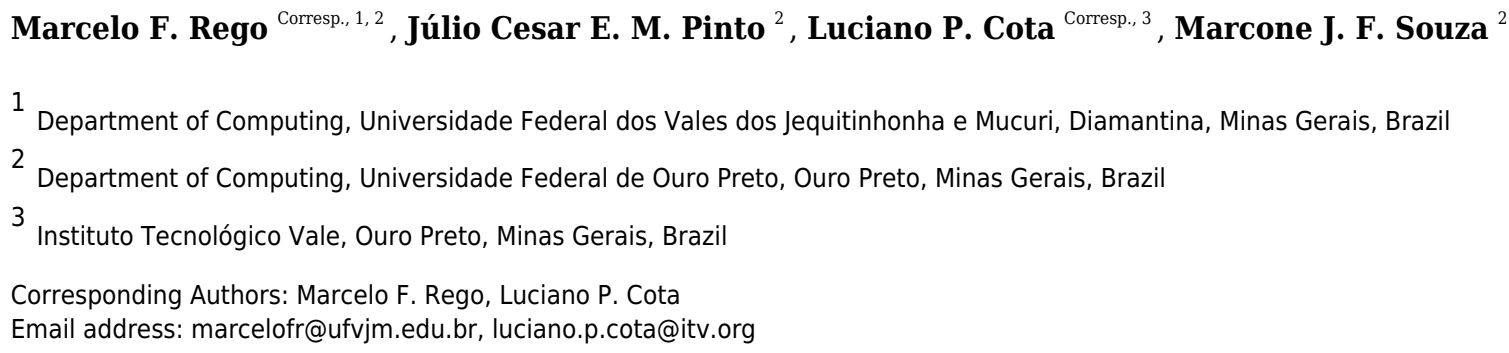

In many countries, there is an energy pricing policy that varies according to the time-ofuse. In this context, it is financially advantageous for the industries to plan their production considering this policy. This article introduces a new bi-objective unrelated parallel machine scheduling problem with sequence-dependent setup times, in which the objectives are to minimize the makespan and the total energy cost. We propose a mixedinteger linear programming formulation based on the weighted sum method to obtain the Pareto front. We also developed an NSGA-II method to address large instances of the problem since the formulation cannot solve it in an acceptable computational time for decision-making. The results showed that the proposed NSGA-II is able to find a good approximation for the Pareto front when compared with the weighted sum method in small instances. Besides, in large instances, NSGA-II outperforms, with 951\% confidence level, the MOGA and NSGA-I multi-objective techniques concerning the hypervolume and hierarchical cluster counting metrics. Thus, the proposed algorithm finds non-dominated solutions with good convergence, diversity, uniformity, and amplitude. 


\title{
A mathematical formulation and an NSGA-II algorithm for minimizing the makespan and energy cost under time-of-use electricity price in an unrelated parallel machine scheduling
}

\author{
Marcelo Ferreira Rego ${ }^{1,2}$, Júlio Cesar Evaristo Moreira Pinto ${ }^{1}$, Luciano
} Perdigão Cota $^{3}$, and Marcone Jamilson Freitas Souza ${ }^{1}$

${ }^{1}$ Department of Computing, Universidade Federal de Ouro Preto, Ouro Preto, MG, 35400-000, Brazil

${ }_{10}^{2}$ Department of Computing, Universidade Federal dos Vales dos Jequitinhonha e Mucuri, Diamantina, MG 39100-000, Brazil

${ }^{3}$ Instituto Tecnológico Vale, Ouro Preto, MG, 354000-000, Brazil

Corresponding author:

Marcelo Ferreira Rego, Luciano Perdigão Cota

15 Email address: marcelofr@ufvjm.edu.br, luciano.p.cota@itv.org

\begin{abstract}
In many countries, there is an energy pricing policy that varies according to the time-of-use. In this context, it is financially advantageous for the industries to plan their production considering this policy. This article introduces a new bi-objective unrelated parallel machine scheduling problem with sequence-dependent

20 setup times, in which the objectives are to minimize the makespan and the total energy cost. We propose a mixed-integer linear programming formulation based on the weighted sum method to obtain the Pareto front. We also developed an NSGA-II method to address large instances of the problem since the formulation cannot solve it in an acceptable computational time for decision-making. The results showed that the proposed NSGA-II is able to find a good approximation for the Pareto front when compared with

25 the weighted sum method in small instances. Besides, in large instances, NSGA-II outperforms, with 95\% confidence level, the MOGA and NSGA-I multi-objective techniques concerning the hypervolume and hierarchical cluster counting metrics. Thus, the proposed algorithm finds non-dominated solutions with good convergence, diversity, uniformity, and amplitude.
\end{abstract}

\section{INTRODUCTION}

The industrial sector is one of the largest consumers of energy in the world. According to EIA (2016), this sector consumes around 54\% of the total energy distributed globally.

Among the various forms of energy used by the manufacturing industry, electricity has been one of the most consumed. In China, for example, this sector consumes about $50 \%$ of the electricity produced in the country (Liu et al., 2014).

35 In recent years, electricity prices have continuously increased for manufacturing companies in industrialized countries (Willeke et al., 2016). In Norway, the industrial electricity price, including taxes, increased by 47\% between 2017 and 2018 (BEIS, 2020). This increase has an impact on production costs and can reduce the competitiveness of companies. In countries that implement a pricing policy so that the energy price depends on the time-of-use, the reduction of electricity costs can occur through production planning that prioritizes periods when energy is less expensive.

However, few studies address scheduling problems in which the energy price depends on the timeof-use tariffs. Among them, we mention Ebrahimi et al. (2020), Zeng et al. (2018), Wang et al. (2016), 
Shrouf et al. (2014), and (Zhang et al., 2014), where the objective includes to minimize the total energy cost.

On the other hand, among several scheduling environments, the unrelated parallel machine one has received much attention recently, given its wide applicability in the industry (Cota et al., 2019). In terms of performance measures, makespan minimization is one of the most common because this criterion aims at the good utilization of the machines (Pinedo, 2016). Lastly, the sequence-dependent setup times appear in many industrial and service applications (Kopanos et al., 2009). However, as far as we know, no work reported in the literature addresses the unrelated parallel machine scheduling problem with sequence-dependent setup times (UPMSP-SDS), considering minimizing the makespan and the total energy cost. This paper, therefore, aims to fill this gap.

The main contributions of this work are the following: $i$ ) introducing a new bi-objective unrelated parallel machine scheduling problem; $i$ ) introducing a new mixed-integer linear programming formulation able to solve small-scale instances of this problem; iii) proposing an adapted version of the NSGA-II algorithm to treat large-scale instances of this problem; $i v$ ) creating a set of instances for this problem; $v$ ) performing an experimental study of the proposed methods.

We organized the remainder of this article as follows: In Section 2, we review the literature. In Section 3, we detail the problem addressed. In Section 4, we introduce the proposed mathematical model. In Section 5, we show the adaptation of the NSGA-II algorithm to the problem. In Section 6, we report the computational results, which include a comparison of the results of the proposed algorithm with the exact method on small instances and a comparison with other multi-objective algorithms on large instances. Finally, we present the conclusions and directions for future work in Section 7.

\section{LITERATURE REVIEW}

Here, we present a literature review with previous research that addressed scheduling problems and considered objectives related to this work.

Some studies address the scheduling problem only to minimize energy consumption. For example, Shrouf et al. (2014) proposed a mathematical model for the scheduling problem on a single machine. However, the model cannot solve large instances within a reasonable computational time for decisionking. For this reason, they also proposed a genetic algorithm. The computational results indicated the possibility of reducing energy consumption by up to $30 \%$ when they compared the genetic algorithm solution and the "as soon as possible" heuristic solution. Tsao et al. (2020) presented a fuzzy model integrated into a genetic algorithm for a problem similar to that described previously. They tested their method in instances of up to 200 jobs. The results indicated a $4.20 \%$ reduction in total energy consumption compared to the traditional genetic algorithm.

Other studies address the scheduling problem and consider a second objective beyond minimizing energy consumption. Cota et al. (2018) proposed a mathematical model and applied a mathematical heuristic called multi-objective smart pool search for the UPMSP-SDS. The objective functions are to minimize the makespan and the total energy consumption. In the experiments, they used a set of so instances with up to fifteen jobs, and five machines randomly generated. They adopted hypervolume and set coverage metrics to compare the proposed algorithm with the $\varepsilon$-constraint exact method. They showed that the objectives are conflicting and that energy consumption strongly influences the solution's quality. Cota et al. (2019) introduced the MO-ALNS and MO-ALNS/D algorithms to handle instances of up to 250 jobs and 30 machines of the same problem described previously. The MO-ALNS algorithm is a multi-objective version of the Adaptive Large Neighborhood Search - ALNS (Ropke and Pisinger, 2006), and MO-ALNS/D combines the multi-objective MOEA/D (Zhang and Li, 2007) with ALNS. The MO-ALNS/D algorithm was able to find better results than MO-ALNS in most instances in the hypervolume, set coverage, and Hierarchical Cluster Counting (HCC) (Guimaraes et al., 2009) metrics. Wu and Che (2019) proposed a memetic differential evolution (MDE) algorithm for the UPMSP in which

90 the objectives are also to minimize the makespan and the total energy consumption. The computational results showed that the proposed approach provides a significant improvement over the basic DE. Also, the MDE outperforms the SPEA-II and NSGA-II algorithms. Liang et al. (2015) presented the Ant Colony Optimization algorithm with the Apparent Tardiness Cost (ACO-ATC) rule for the UPMSP seeking to minimize the total tardiness and the energy consumption. In this problem, machines need to wait until jobs are ready. However, it is necessary to decide whether the machine remains on or off during the wait. Turning off the machine to wait for the job to be ready saves energy. On the other hand, keep on 
the machine while waiting for the job saves time because it eliminates the need to prepare the machine again. They compared the ACO-ATC results with the classic ACO and a GRASP-based algorithm (Feo and Resende, 1995). The proposed algorithm was better than the other approaches in most of the tested instances.

There are studies that only address the minimization of the total energy cost. Ding et al. (2016) presented two approaches to UPMSP: the first introduces a time-interval-based Mixed Integer Linear Programming (MILP) formulation. The second consists of a reformulation of the problem using the Dantzig-Wolfe decomposition and a column generation heuristic. According to the results, the MILP formulation overcame the column generation method in terms of solution quality and execution time when electricity prices stay stable for a relatively long period. On the other hand, the column generation method performed better when the electricity price frequently changed (i.e., every half hour). Cheng et al. (2018) improved the formulation by Ding et al. (2016) and performed computational experiments with 120 randomly generated instances to compare the two formulations. The results showed that the new formulation achieves better results concerning the solution quality and execution time. Saberi-Aliabad et al. (2020) proposed the fix-and-relax heuristic algorithm in two stages for this same problem. In the first stage, jobs are assigned to the machines, and the second one solves a scheduling problem on simple machines. They tested its method in 20 instances randomly generated following the same parameter values as other previous studies. They compared the proposed method with the algorithms of Che et al. (2017) and Cheng et al. (2018). The results showed that the fix-and-relax algorithm overcame the others.

Finally, we present studies that address the scheduling problem considering minimizing the energy cost combined with another objective. Zeng et al. (2018) dealt with the bi-objective uniform parallel machine scheduling to minimize the total energy cost and the number of machines. They proposed a new mathematical model and a heuristic algorithm for it. The computational results showed that the uristic method generates high-quality solutions in a reasonable time limit for instances of up to 5000 jobs. Cheng et al. (2019) presented a mathematical formulation and a genetic algorithm for the UPMSP. They considered the objective of minimizing the weighted sum of makespan and total electricity cost. The results presented by their formulation overcome that of the genetic algorithm in terms of solution quality. Kurniawan et al. (2017) proposed a genetic algorithm with a delay mechanism for the UPMSP to minimize the weighted sum of makespan and total energy cost. The proposed algorithm handled instances of up to 30 jobs and 15 machines. The results showed that the proposed method provided better solutions than the classical genetic algorithm.

Although there are studies correlated to ours, of our knowledge, there is no work addressing the unrelated parallel machine scheduling problem with sequence-dependent setup times to minimize the makespan and the total energy cost. Table 1 summarizes the characteristics of scheduling problems treated by our work compared to literature references.

Table 1. Summary of characteristics addressed by our work compared to literature studies.

\begin{tabular}{|c|c|c|c|c|c|c|c|c|}
\hline Reference & $\begin{array}{l}\text { Unrelated } \\
\text { parallel } \\
\text { machines }\end{array}$ & $\begin{array}{l}\text { Sequence- } \\
\text { dependent } \\
\text { setup }\end{array}$ & Makespan & $\begin{array}{l}\text { Total } \\
\text { energy } \\
\text { cost }\end{array}$ & $\begin{array}{l}\text { time- } \\
\text { of- } \\
\text { use }\end{array}$ & $\begin{array}{l}\text { Multi- } \\
\text { objective }\end{array}$ & $\begin{array}{l}\text { Exact } \\
\text { method }\end{array}$ & $\begin{array}{l}\text { Metaheuristic } \\
\text { method }\end{array}$ \\
\hline Shrouf et al. (2014) & & & & $\checkmark$ & & & $\checkmark$ & $\checkmark$ \\
\hline Liang et al. (2015) & $\checkmark$ & & & & & $\checkmark$ & $\checkmark$ & $\checkmark$ \\
\hline Ding et al. (2016) & $\checkmark$ & & & $\checkmark$ & $\checkmark$ & & $\checkmark$ & \\
\hline Kurniawan et al. (2017) & $\checkmark$ & & $\checkmark$ & $\checkmark$ & $\checkmark$ & $\checkmark$ & & $\checkmark$ \\
\hline Cota et al. (2018) & $\checkmark$ & $\checkmark$ & $\checkmark$ & & & $\checkmark$ & $\checkmark$ & \\
\hline Cheng et al. (2018) & $\checkmark$ & & & $\checkmark$ & $\checkmark$ & & $\checkmark$ & \\
\hline Zeng et al. (2018) & & & & $\checkmark$ & $\checkmark$ & $\checkmark$ & $\checkmark$ & $\checkmark$ \\
\hline Cota et al. (2019) & $\checkmark$ & $\checkmark$ & $\checkmark$ & & & $\checkmark$ & & $\checkmark$ \\
\hline Wu and Che (2019) & $\checkmark$ & & $\checkmark$ & & & $\checkmark$ & & $\checkmark$ \\
\hline Cheng et al. (2019) & $\checkmark$ & & $\checkmark$ & $\checkmark$ & $\checkmark$ & $\checkmark$ & $\checkmark$ & \\
\hline Tsao et al. (2020) & & & & $\checkmark$ & & & $\checkmark$ & $\checkmark$ \\
\hline Saberi-Aliabad et al. (2020) & $\checkmark$ & & & $\checkmark$ & $\checkmark$ & & $\checkmark$ & $\checkmark$ \\
\hline Our proposal & $\checkmark$ & $\checkmark$ & $\checkmark$ & $\checkmark$ & $\checkmark$ & $\checkmark$ & $\checkmark$ & $\checkmark$ \\
\hline
\end{tabular}

\section{PROBLEM STATEMENT}

To define the UPMSP-SDS, we characterize the problem in this section and introduce a MILP formulation to solve it.

The following are the characteristics of the problem addressed in this work: 
- There are a set $N=\{1, \ldots, n\}$ of jobs, a set $M=\{1, \ldots, m\}$ of machines, and a set $L=\{1, \ldots, o\}$ of different operation modes, such that each operation mode $l \in L$ is associated with a multiplication factor of speed $v_{l}$ and a multiplication factor of power $\lambda_{l}$;

- The machines are unrelated parallel. In other words, the processing time of job $j \in N$ can be different on each machine $i \in M$;

- There is a planning horizon that consists of a set of $H=\{0, \ldots,|H|\}$ of time instants, and we must perform all jobs within this horizon;

- All jobs are available to be processed at the beginning of the planning horizon $h=0$;

- Each job $j \in N$ must be allocated to exactly one machine $i \in M$;

- There is a processing time $p_{i j}$ to process a job $j \in N$ on a machine $i \in M$;

- There is a sequence-dependent setup time $S_{i j k}$ to execute a job $k \in N$ after another job $j \in N$ on a machine $i \in M$;

- Each machine $i \in M$ has a power $\pi_{i}$ at normal operating speed;

- The operation mode $l \in L$ of each job determines the multiplication factor of power $\left(\lambda_{l}\right)$. It also determines the multiplication factor of speed $\left(v_{l}\right)$, which, in turn, is related to the execution time of each job;

- There is a set $D$ of days on the planning horizon $H$;

- Each day is discretized into sizeD time intervals. For example, for discretizing a day in minutes, size $D=1440$; for the discretization of one day in hours, size $D=24$;

- To each day $t \in H$, we have a peak hour, which starts at the time start $p_{t} \in H$ and ends at the time end $p_{t} \in H$;

- $E T^{\text {off }}$ and $E T^{o n}$ represent the energy tariff $(\$ / \mathrm{KWh})$ in off-peak hours and on-peak hours, respectively.

Table 2 presents the decision and auxiliary variables that are needed to model the problem.

Table 2. Decision and auxiliary variables for the problem

\begin{tabular}{l|l}
\hline Name & Description \\
\hline$X_{i j h l}$ & Binary variable that assumes value 1 if the job $j$ is allocated on the machine $i$ \\
$P E C_{t}^{o n}$ & at time $h$ and in the operation mode $l$, and value 0, otherwise \\
$P E C_{t}^{\text {off }}$ & Partial Energy Cost (\$) during the on-peak in day $t \in D$ \\
$C_{\max }$ & Partial Energy Cost (\$) during the off-peak in day $t \in D$ \\
$T E C$ & The maximum completion time of the jobs, also known as makespan \\
\hline
\end{tabular}

Thus, we can define the problem through Equations (1) - (12).

$\min C_{\max }$

$\min T E C$ 
Subject to:

$$
\begin{aligned}
& \sum_{i=1}^{m} \sum_{l=1}^{o} \sum_{h=0}^{|H|-\left[\frac{P_{i j}}{V_{l}}\right\rceil} X_{i j h l}=1 \quad \forall j \in N \\
& X_{i j h l}+\sum_{u=h}^{\min \left(h+\left[\frac{P_{i j}}{V_{l}}\right]+S_{i j k}-1,|H|\right)} \sum_{l_{1}=1}^{o} X_{i k u l_{1}} \leq 1 \quad \forall i \in M, j \in N, k \in N, l \in L, j \neq k \\
& C_{\max } \geq X_{i j h l} \times\left[h+\left\lceil\frac{P_{i j}}{V_{l}}\right]\right], \quad \forall i \in M, j \in N, h \in H, l \in L \\
& P E C_{t}^{o n} \geq \sum_{i=1}^{m} \sum_{j=1}^{n} \sum_{l=1}^{o} \frac{\lambda_{l} \times \pi_{i} \times E T^{o n} \times 24}{s i z e D} \times \\
& \left\{\sum_{h=s i z e D \times(t-1)}^{\text {start }_{t}-1} X_{i j h l} \times\right. \\
& {\left[\max \left(0, \min \left(h+\left\lceil\frac{P_{i j}}{V_{l}}\right\rceil-1, \text { end } p_{t}\right)-\left(\text { start }_{t}-1\right)\right)\right]} \\
& \left.+\sum_{h=\text { start } p_{t}}^{\text {end } p_{t}-1} X_{i j h l} \times\left[\min \left(h+\left\lceil\frac{P_{i j}}{V_{l}}\right\rceil, \text { end } p_{t}+1\right)-h\right]\right\} \quad \forall t \in D
\end{aligned}
$$$$
P E C_{t}^{o f f} \geq \sum_{i=1}^{m} \sum_{j=1}^{n} \sum_{l=1}^{o} \frac{\lambda_{l} \times \pi_{i} \times E T^{o f f} \times 24}{s i z e D} \times
$$

$\left\{\sum_{h=s i z e D \times(t-1)}^{\text {startp }_{t}-1} X_{i j h l} \times\right.$

$\left\lceil\min \left(h+\left\lceil\frac{P_{i j}}{V_{l}}\right\rceil\right.\right.$, start $\left.p_{t}\right)-h+\max \left(0, h+\left\lceil\frac{P_{i j}}{V_{l}}\right\rceil-\right.$ end $\left.\left.p_{t}-1\right)\right]$

$+\sum_{h=\text { start } p_{t}}^{\text {end } p_{t}-1} X_{i j h l} \times\left[\max \left(0, h+\left\lceil\frac{P_{i j}}{V_{l}}\right\rceil-\right.\right.$ end $\left.\left.p_{t}-1\right)\right]$

$\left.+\sum_{h=e n d p_{t}}^{|H|-1} X_{i j h l} \times\left\lceil\frac{P_{i j}}{V_{l}}\right\rceil\right\}$

$\forall t \in D$

$T E C \geq \sum_{t=1}^{\text {sizeD }}\left(P E C_{t}^{o f f}+P E C_{t}^{o n}\right)$

$X_{i j h l} \in\{0,1\}$

$\forall i \in M, j \in N, h \in H, l \in L$

$C_{\max } \geq 0$

$P E C_{t}^{\text {off }} \geq 0$

$\forall t \in D$

$P E C_{t}^{o n} \geq 0$

$\forall t \in D$

The objectives of the problem are to minimize, simultaneously, the makespan and the total energy cost, defined by Equations (1) and (2), respectively. The set of constraints (3) ensures that every job $j \in J$ is allocated on a machine has a single operation mode, and ends its execution inside the planning horizon. Constraints (4) define that if the job $k$ is assigned to the machine $i$ immediately after the job $j$, then the start time of the job $k$ must be greater than the sum of the end time of the job $j$ and the setup time between them. It is important to highlight that for the previous model to be valid, the setup and processing times must satisfy the triangular inequality, as defined by Rosa and Souza (2009). The set of constraints (5) determines a lower bound for the makespan. Constraints (6) and (7) define a lower 
bound for the partial energy cost in the on-peak hours $\left(P E C_{o n}\right)$ and the total energy cost for off-peak hours $\left(P E C_{o f f}\right)$, respectively. It is important to note that a job can be partially performed in the on-peak hours and partially in the off-peak hours and that the total energy cost is directly related to the energy price and the job execution time. Constraint (8) ensures a lower bound for the total energy cost. Constraints (9)-(12) define the domain of the decision and auxiliary variables of the problem.

The calculation of the energy cost of a job $j$ depends on its execution time during the on-peak and off-peak time. Thus, there are six possible cases:

Case 1: The job $j$ starts and ends before the on-peak hours;

Case 2: The job $j$ starts before the on-peak hours and ends in the on-peak hours;

Case 3: The job $j$ starts and ends in the on-peak hours;

Case 4: The job $j$ starts during the on-peak hours and ends after the on-peak hours;

Case 5: The job $j$ starts and ends after the on-peak hours;

Case 6: The job $j$ starts before the on-peak hours and ends after the on-peak hours.

To illustrate cases 1 to 5, let Figure 1. It shows the execution of five jobs $N=\{2,4,1,5,3\}$ in the scheduling of a single machine $i=1$ in a single operation mode $l=1$ on day $t=1$ of the planning horizon. Let the start of the on-peak hours (start $p_{1}$ ) equal to 18 ; the end of the on-peak hours (end $\left.p_{1}\right)$ equal to 21 ; the multiplication factor of power $\left(\lambda_{l}\right)$ equal to 1 ; the energy consumption of machine at normal operation $\left(\pi_{1}\right)$ equal to 100 ; the energy tariff in the on-peak hours $\left(E T^{o n}\right)$ equal to $0.10 \$ / K W h$ and in the off-peak hours $\left(E T^{o f f}\right)$ equal to $0.05 \$ / K W h$; the multiplication factor of speed $v_{l}$ equal to 1 . In this example, we consider discretization in hours. This figure shows that jobs 4,1 , and 5 are performed in the on-peak hours, partially or totally, and jobs 2 and 3 , in turn, in the off-peak hours.

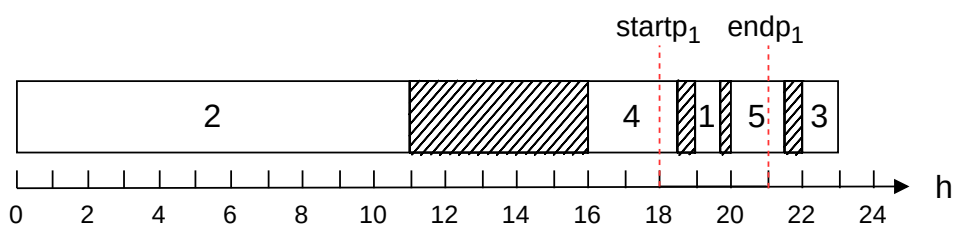

Figure 1. Example to illustrate the calculation of the energy cost on a machine

$$
\begin{aligned}
& P E C_{1}^{o n}=\underbrace{\sum_{j=1}^{n} \frac{1 \times 100 \times 0.10 \times 24}{24} \times}_{\text {Parcel 1(a) }} \\
& \underbrace{\left\{\sum_{h=0}^{18-1} X_{1 j h 1} \times\left[\max \left(0, \min \left(h+\left[\frac{P_{1 j}}{1}\right]-1,21\right)-(18-1)\right)\right]\right.}_{\text {Parcel } 2(\mathrm{~b})} \\
& +\underbrace{\left.\sum_{h=18}^{21-1} X_{1 j h 1} \times\left[\min \left(h+\left[\frac{P_{1 j}}{1}\right], 21+1\right)-h\right]\right\}}_{\text {Parcel }}
\end{aligned}
$$




$$
\begin{aligned}
& P E C_{1}^{o f f}=\underbrace{\sum_{j=1}^{n} \frac{1 \times 100 \times 5 \times 24}{24}}_{\text {Parcel } 1(\mathrm{~d})} \times \\
& \underbrace{\left\{\sum_{h=0}^{18-1} X_{1 j h 1} \times\left[\min \left(h+\left\lceil\frac{P_{1 j}}{1}\right\rceil, 18\right)-h+\max \left(0, h+\left\lceil\frac{P_{1 j}}{1}\right\rceil-21-1\right)\right]\right.}_{\text {Parcel 2(e) }} \\
& +\underbrace{\sum_{h=18}^{21-1} X_{1 j h 1} \times\left[\max \left(0, h+\left\lceil\frac{P_{1 j}}{1}\right\rceil-21-1\right)\right]} \\
& \text { Parcel 3(f) } \\
& +\underbrace{\left.\sum_{h=21}^{24-1} X_{1 j h 1} \times\left\lceil\frac{P_{1 j}}{1}\right\rceil\right\}}_{\text {Parcel } 4(\mathrm{~g})}
\end{aligned}
$$

Table 3 illustrates the contribution of each job to the total energy cost, according to the example in Figure 1. The column "\# Job" represents the job, the column "Case" shows the contemplated case, and the columns "Contr. on-peak" and "Contr. off-peak" show the contributions of the job to the energy cost of each job in the on-peak and off-peak hours, respectively.

Table 3. Energy cost by job in the Example of Figure 1

\begin{tabular}{c|c|c|c} 
\# Job & Case & Contr. on-peak hours & Contr. off-peak hours \\
\hline 2 & 1 & $\underbrace{10}_{\text {(a) }} \times(\underbrace{0}_{(\mathrm{b})}+\underbrace{0}_{(\mathrm{c})})=0$ & $\underbrace{5}_{(\mathrm{d})} \times(\underbrace{11}_{(\mathrm{e})}+\underbrace{0}_{(\mathrm{f})}+\underbrace{0}_{(\mathrm{g})})=55$ \\
\hline 4 & 2 & $\underbrace{10}_{(\mathrm{a})} \times(\underbrace{1}_{(\mathrm{b})}+\underbrace{0}_{(\mathrm{c})})=10$ & $\underbrace{5}_{(\mathrm{d})} \times(\underbrace{2}_{(\mathrm{e})}+\underbrace{0}_{(\mathrm{f})}+\underbrace{0}_{(\mathrm{g})})=10$ \\
\hline 1 & 3 & $\underbrace{10}_{(\mathrm{a})} \times(\underbrace{0}_{(\mathrm{b})}+\underbrace{1}_{(\mathrm{c})})=10$ & $\underbrace{5}_{(\mathrm{d})} \times(\underbrace{0}_{(\mathrm{e})}+\underbrace{0}_{(\mathrm{f})}+\underbrace{0}_{(\mathrm{g})})=0$ \\
\hline 3 & 4 & $\underbrace{10}_{(\mathrm{a})} \times(\underbrace{0}_{(\mathrm{b})}+\underbrace{1}_{(\mathrm{c})})=10$ & $\underbrace{5}_{(\mathrm{d})} \times(\underbrace{0}_{(\mathrm{e})}+\underbrace{1}_{(\mathrm{f})}+\underbrace{0}_{(\mathrm{g})})=5$ \\
\hline
\end{tabular}

The total energy cost found to the schedule shown in Figure 1 is 105 .

To illustrate case 6, consider Figure 2. It shows the execution of three jobs $N=\{2,1,3\}$ on a single machine $i=1$ in operating mode $l=1$ during day $t=1$ of the planning horizon. Let also the start of the on-peak hours $\left(\right.$ start $\left._{1}\right)$ equal to 18 ; the end of the on-peak hours ( $\left.e n d p_{1}\right)$ equal to 21 ; the multiplication factor of power $\left(\lambda_{l}\right)$ equal to 1 ; the energy consumption of machines at normal operation $\left(\pi_{1}\right)$ equal to 100 ; the energy tariff in the on-peak hours $\left(E T^{o n}\right)$ equal to $0.10 \$ / K W h$ and in the off-peak hours $\left(E T^{o f f}\right)$ equal to $0.05 \$ / K W h$; and the multiplication factor of speed equal to 1 . Such as in the previous example, we consider discretization in hours. This figure shows that job 1 is performed in the on-peak hours and jobs 2 and 3, in turn, in the off-peak hours.

The contribution of the job 1 to the energy cost in the on-peak hours is 30, and the contribution to the cost in the off-peak hours is 50 .

Thus, calculating similarly to the previous example, we conclude that the total energy cost for the schedule shown in Figure 2 is 155.

\section{WEIGHTED SUM METHOD}

We used the weighted sum method (Marler and Arora, 2004) to solve the multi-objective optimization 


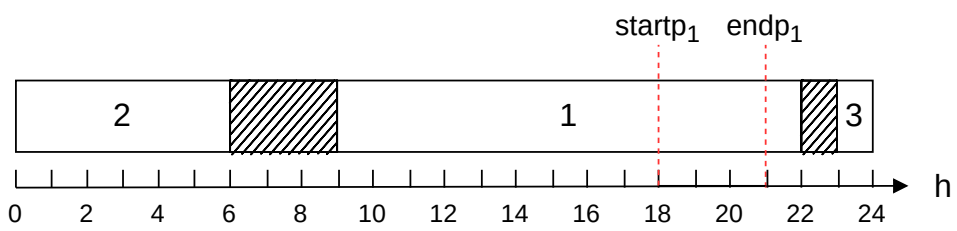

Figure 2. Schedule example for case 6

For this, consider Equation (15):

$$
\min z(X)=\left[\alpha \times\left(\frac{C_{\max }}{|H|}\right)+(1-\alpha) \times\left(\frac{T E C}{\text { Cost }_{\max }}\right)\right]
$$

where:

- $\alpha$ : real number in range $[0,1]$;

- $|H|$ : represents the cardinality of the set $H$;

- $\operatorname{Cost}_{\max }$ : is the estimate for the maximum energy cost used to normalize the total energy cost. It is calculated using a heuristic, as shown in Section 5.1;

The problem constraints are those defined by Equations (3)-(12).

Algorithm 1 describes all the steps of the weighted sum method implemented.

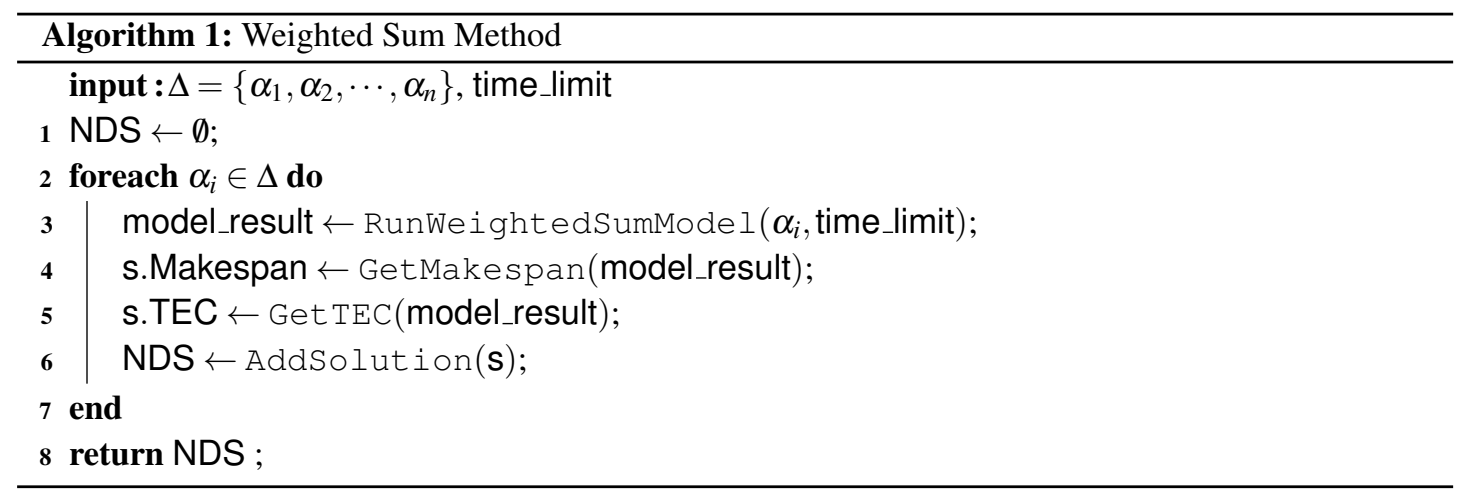

Algorithm 1 receives as input: the set $\Delta$ with the values for $\alpha$ and the time limit. In line 1 , we initialize the non-dominated set (NDS) as empty. Then, we execute the loop defined between lines 2-7 for each value $\alpha$. In line 3, we obtain the result from the execution of the model. Then, we get the Makespan and TEC values resulting from the model execution. Then, in line 6 , we add the solution obtained to the NDS. Finally, in line 8, the method returns the generated non-dominated set.

225 In the weighted sum method, the decision-maker must define a weight for each objective function. The value of this weight reflects the relative importance of each objective in the overall solution. We adopted several combinations of weights to find the most significant number of optimal Pareto solutions to the problem addressed.

We used the following parameters for Algorithm 1:

- The set $\Delta=\{0,0.1,0.2,0.3,0.4,0.5,0.6,0.7,0.8,0.9,1\}$ with the possible values for $\alpha$;

- The time limit for each execution of the mathematical model, defined as time_limit $=800 \times n \times$ $\ln (m)$ seconds for each $\alpha$ value, where $m$ is the number of machines and $n$ is the number of jobs;

- $\operatorname{size} D=144$ : to discretize the day at intervals of 10 minutes each. 


\section{PROPOSED NSGA-II}

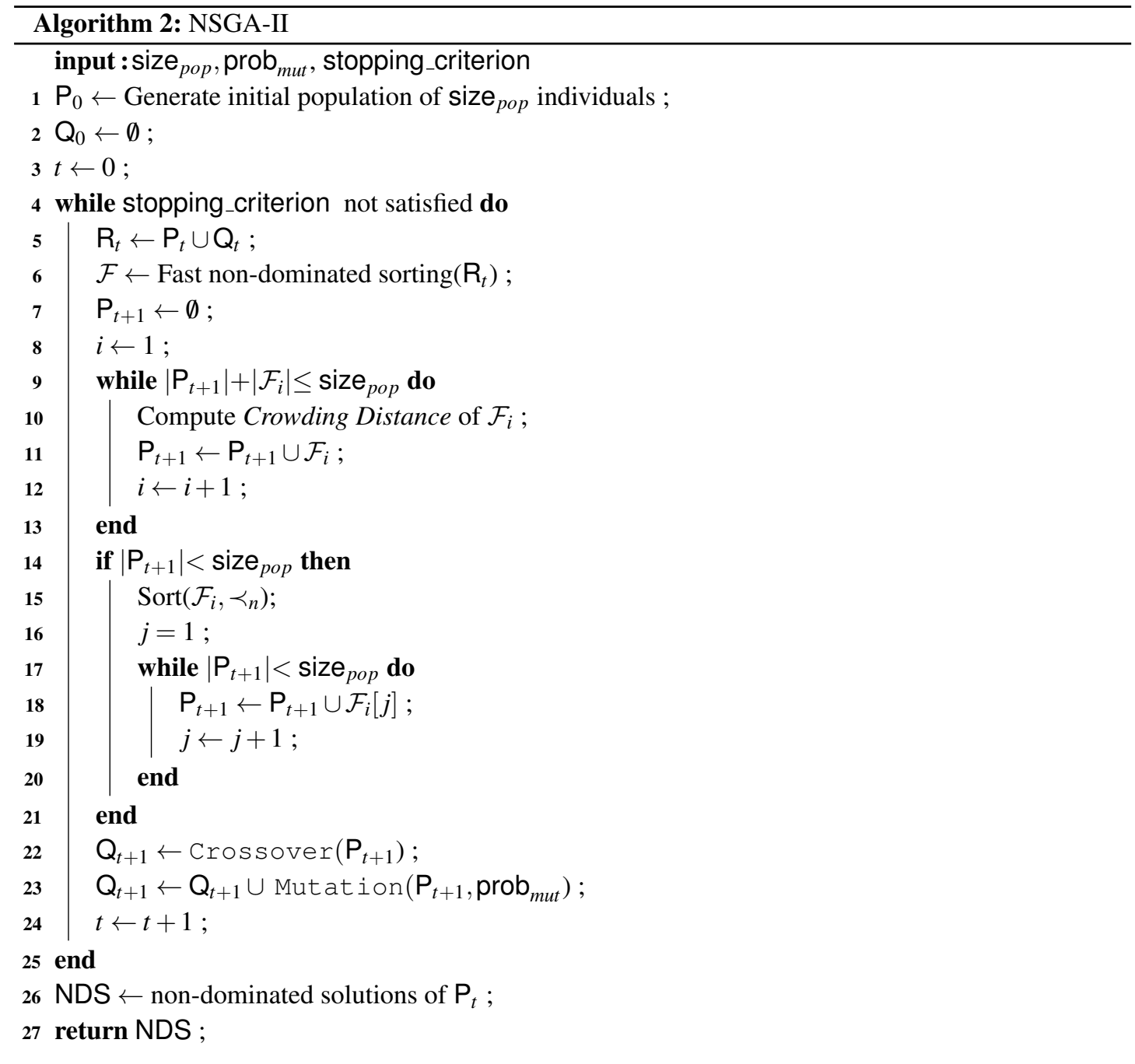

Algorithm 2 receives the following input parameters: the population size $\left(\right.$ size $\left._{\text {pop }}\right)$, the probability of mutation ( prob $_{m u t}$ ), and the stopping_criterion. In line 2, we created an initial population $P_{0}$. Then, in the main loop (lines 4-25), we combine the parent $P_{t}$ and offspring population $Q_{t}$ to generate a new population $\mathrm{R}_{t}$ (line 5). In line 6 , we apply the fast non-dominated sorting method to divide the population $\mathrm{R}_{t}$ into non-dominated sets, called fronts, $\mathcal{F}_{1}, \mathcal{F}_{2}, \ldots, \mathcal{F}_{k}$. A front $\mathcal{F}_{i}$ dominates another $\mathcal{F}_{j}$, if and only if, $i<j$ and $\mathrm{R}_{t}=\mathcal{F}_{1} \cup \mathcal{F}_{2} \ldots \mathcal{F}_{k}$. In lines 9-13, we select the best frontiers of $\mathcal{F}$ to include in the population $\mathrm{P}_{t+1}$. We repeat this procedure as long as it is possible to include a new frontier in $\mathrm{P}_{t+1}$ without exceeding the population size. Then we check the size of the population obtained. If it is not exactly size $e_{p o p}$, then we order the next frontier $i$ of $\mathcal{F}$ that has not yet been included in $\mathrm{P}_{t+1}$, according to the crowding distance, and we select the size $e_{p o p}-\left|\mathrm{P}_{t+1}\right|$ first to fill all spaces of the population $\mathrm{P}_{t+1}$. In lines 22 and 23 , we apply the crossover and mutation operators in $P_{t+1}$ to generate the population $Q_{t+1}$. 
The following subsections describe how an initial population is generated and the crossover and mutation operators, respectively.

\subsection{Initial Population}

The initial population of the NSGA-II contains size ${ }_{p o p}$ individuals. Two of them are constructed through a greedy strategy, one of which considers only the objective of minimizing the makespan. In this case, we always choose the operation mode related to the highest speed factor. The other individual considers only the total energy cost. In this case, we choose the operation mode related to the lowest consumption factor. The other individuals (size $e_{\text {pop }}-2$ ) of the initial population are randomly generated.

Algorithm 3 describes the greedy strategy for generating an individual to the initial population.

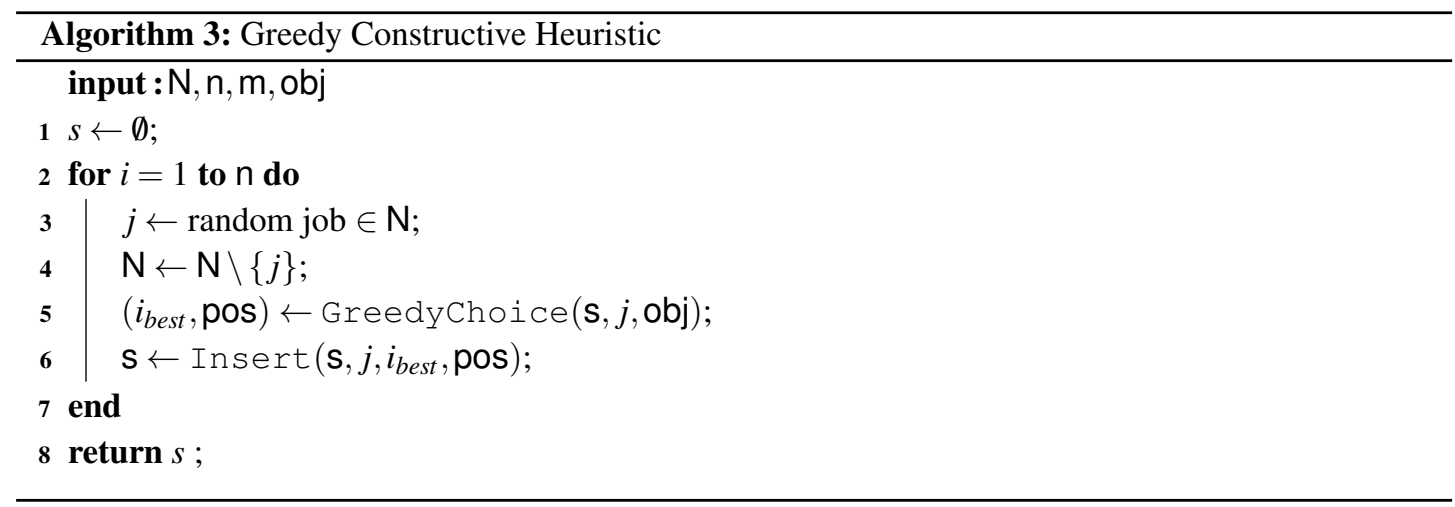

Algorithm 3 starts with an empty initial individual (line 1). The loop between lines 2 and 7 allocates each job $\mathrm{n}$ on the machines. Therefore, first, we randomly select a job $j$, which has not yet been allocated (line 3). Then, we identified in the best machine $i_{\text {best }}$ of the individual $\mathrm{s}$ and the best position pos to insert the job $j$ (line 5). In this case, we consider only one of the objectives of the problem: minimize the makespan or the total energy cost. Then, we allocate job $j$ in position pos of machine $i_{\text {best }}$ in individual $\mathrm{s}$ (line 6). At the end of this procedure, we return a valid individual s (line 8).

\subsection{Crossover}

used the binary tournament selection method to choose each pair of individuals for the crossover operator. We run two tournaments with two individuals each and select the winner of each tournament for the crossover. In our approach, the dominant individual wins the tournament. If both individuals are non-dominated, then we randomly choose an objective and use it to define the winner of the tournament.

Figure 3 illustrates the crossover between two individuals.

After selecting two individuals named parent 1 and parent 2, respectively, we applied the crossover operator to generate new individuals. We adopted the One Point Order Crossover operator from Vallada and Ruiz (2011) adapted to the parallel machine problem. We describe its operation below:

1. We define, at random, the crossover points of each machine, as shown in Figure 3a;

2. We generate two offspring. The first receives the genes to the left of the crossover point defined on each machine of parent 1 . The second receives the genes to the right, as shown in Figure 3b;

3. We mark in parent 2 the genes present in each offspring, as shown in Figure 3c;

4. We add the unmarked genes of parent 2 to offspring 1 and 2. We add these genes in the position that results in the lowest value for the objective function, whereas this problem has two objective functions, so we randomly select one at each crossover. In the end, we will have two new individuals, as shown in Figure 3d.

We repeat this procedure until to generate size $_{p o p}$ new individuals. 

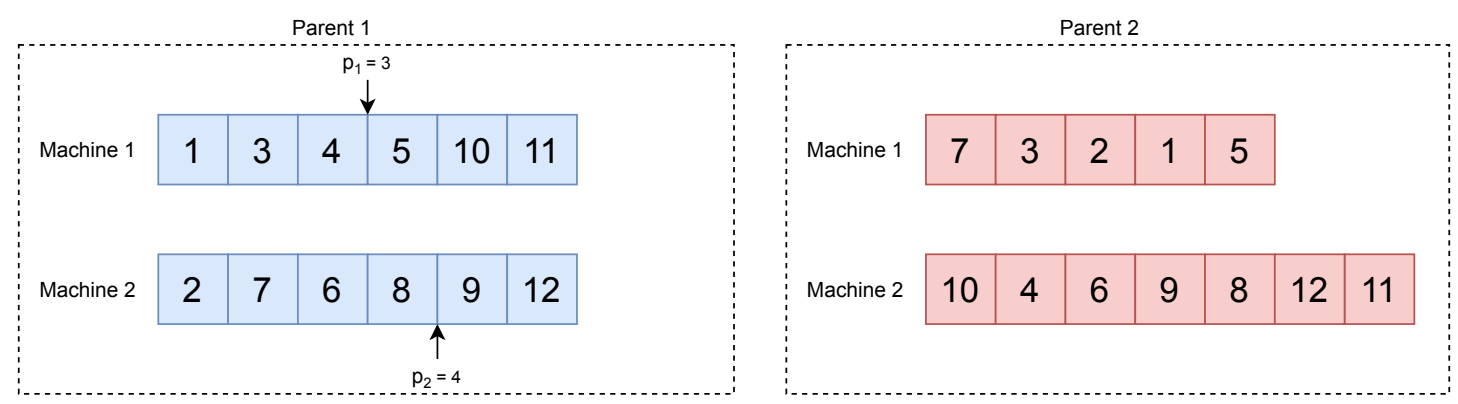

(a) Selecting parents and crossover points
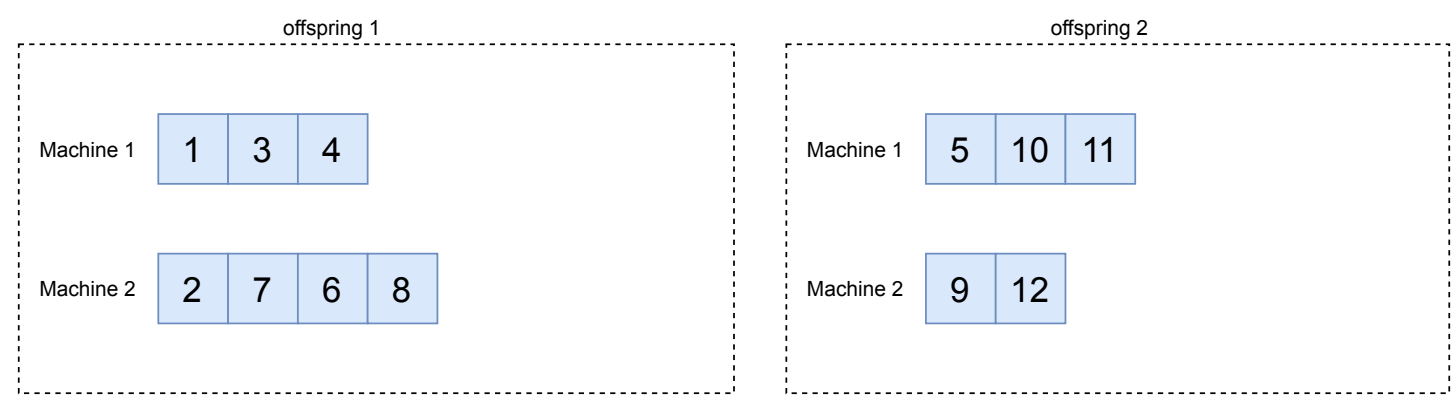

(b) Copy part of genes from parent 1 to each offspring
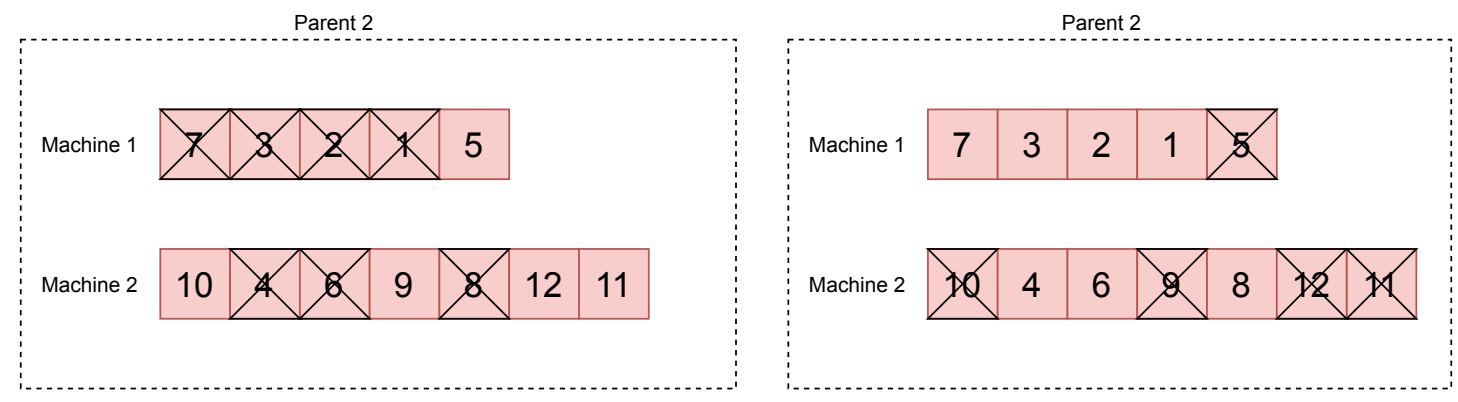

(c) Mark in parent 2 the genes that each offspring already has.
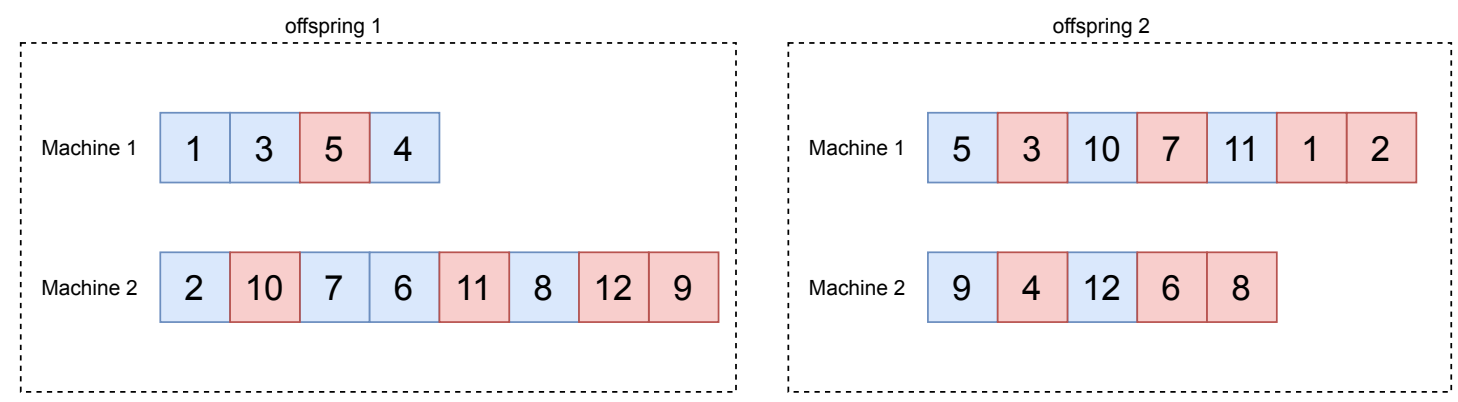

(d) Complete the genes of offspring with the genes of parent 2

Figure 3. Crossover adapted from Vallada and Ruiz (2011)

\subsection{Mutation}

We implemented three mutation operators (Swap, Insert, and Swap of operation mode), described below. These operators maintain the population's genetic diversity and reduce the chances that the algorithm getting stuck at a local optimum. 


\subsubsection{Swap}

The swap operator works by randomly choosing a job $j_{1}$, initially allocated in position $a$ on machine $i_{1}$ and another job $j_{2}$ allocated in position $b$ of machine $i_{2}$. Then, we allocate job $j_{1}$ in position $b$ of machine $i_{2}$, and we allocate job $j_{2}$ in position $a$ of machine $i_{1}$.

Figure 4 illustrates the swap between two jobs $j_{1}$ and $j_{2}$. They are initially allocated on machines $i_{1}$ and $i_{2}$, respectively. After swapping, we allocate job $j_{2}$ on machine $i_{1}$ and job $j_{1}$ on machine $i_{2}$.

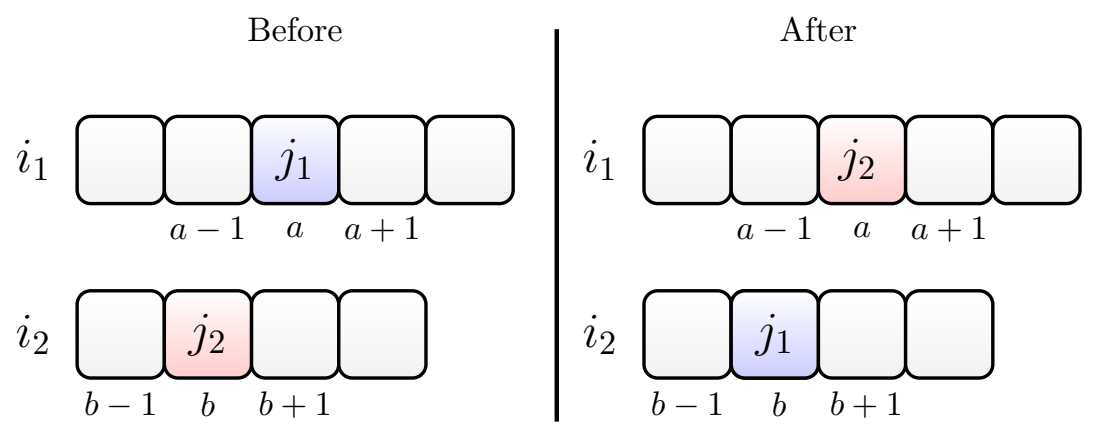

Figure 4. Swap move between jobs $j_{1}$ and $j_{2}$

\subsubsection{Insertion}

The insertion operator consists of randomly choosing a job $j_{1}$ allocated at position $a$ of machine $i_{1}$ and randomly choosing position $b$ of another machine $i_{2}$. Then job $j_{1}$ is removed from machine $i_{1}$ and inserted into position $b$ on the machine $i_{2}$.

Figure 5 illustrates this move. The left side shows the scheduling before, and the right side shows it after the insertion move.

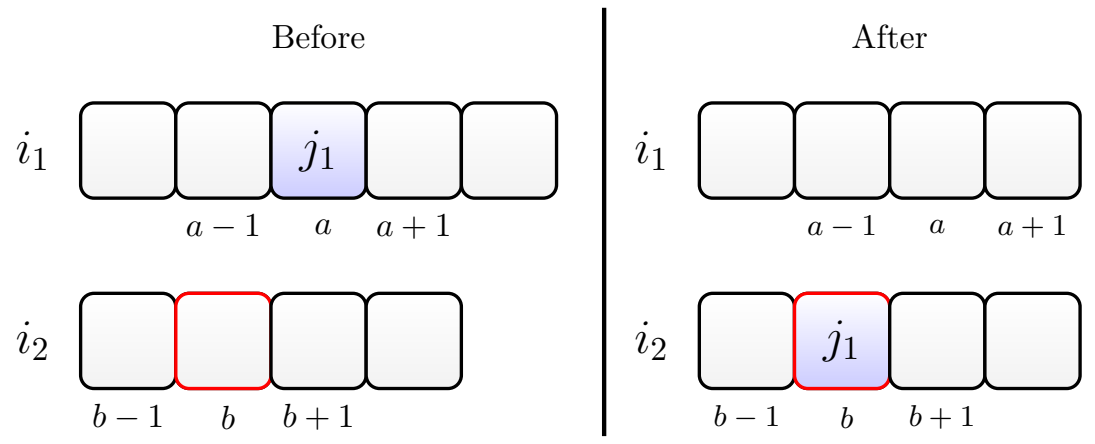

Figure 5. Insertion move of job $j_{1}$ on machine $i_{2}$

\subsubsection{Mode change}

In the operation mode change operator, we randomly select a job and change its operation mode at

Figure 6 illustrates the application of this operator to the scheduling of offspring 1 of Figure 3(d), which involves 12 jobs. As can be seen, job 8, which is in the sixth position of machine 2, has operation mode 3. After the application of this operator, the job changes to operation mode 1.

The NSGA-II algorithm implemented performs a mutation with a probability equal to $\mathrm{prob}_{m u t}$.

\section{COMPUTATIONAL EXPERIMENTS}

We coded the NSGA-II algorithm in the $\mathrm{C}++$ language and implemented the mathematical model with the Gurobi 7.0.2 API (Gurobi Optimization, 2020). We performed the tests on a microcomputer with the 

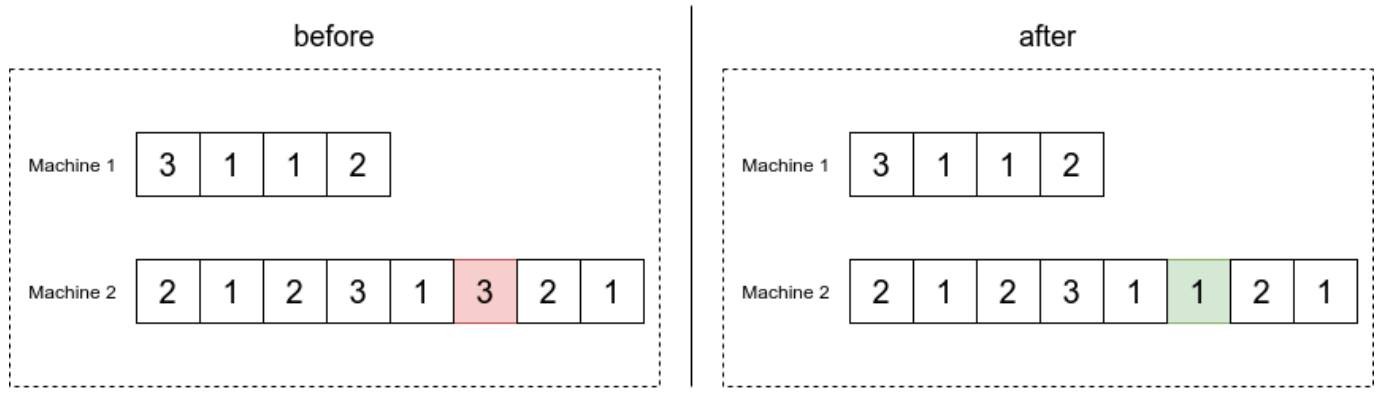

Figure 6. Example of the mode change operator

following configurations: Intel (R) Core (TM) i7-4510U processor with a frequency of $2 \mathrm{GHz}, 16 \mathrm{~GB}$ of RAM, and 64-bits Ubuntu 19.10 operating system.

Furthermore, we compared the performance of the NSGA-II algorithm with two basic multi-objective algorithms: MOGA of Murata et al. (1995) and NSGA-I of Srinivas and Deb (1994). These algorithms use the same NSGA-II crossover and mutation operators described in Subsections 5.2 and 5.3 and the same stopping criterion.

This section is organized as follows. Subsections 6.1 and 6.2 describe the instances and the metrics used to assess the quality of the set of non-dominated solutions generated by the algorithms. Subsection 6.3 shows the parameter calibration of the algorithms. Subsection 6.4 reports the results.

\subsection{Instances Generation}

Since, as far as we know, there is no set of instances in the literature for the problem addressed, we adapted two instance sets from the literature that deal with similar problems. The first one, called set1, is subset of the small instances of Cota et al. (2018) satisfying the triangular inequality, in which we add information about the energy price on-peak and off-peak hours. The second set, named set 2 , is also a subset of the large instances of Cota et al. (2018), in which we included instances of 750 jobs. Table 4 shows the characteristics of these sets of instances, which are are available in Rego et al. (2021).

Table 4. Instance characteristics

\begin{tabular}{|c|c|c|c|}
\hline Parameter & set1 & set2 & Based on \\
\hline$n$ & $6,7,8,9,10$ & $50,250,750$ & Vallada and Ruiz (2011), Cota et al. (2018) \\
\hline$m$ & 2 & 10,20 & Vallada and Ruiz (2011), Cota et al. (2018) \\
\hline$o$ & 3 & 5 & $\begin{array}{l}\text { Mansouri et al. (2016), Ahilan et al. } \\
\text { (2013), Cota et al. (2018) }\end{array}$ \\
\hline$P_{i j}$ & $U[1,99]$ & $U[1,99]$ & Vallada and Ruiz (2011), Cota et al. (2018) \\
\hline$S_{i j k}$ & $U[1,9]$ & $U[1,9], U[1,124]$ & Vallada and Ruiz (2011), Cota et al. (2018) \\
\hline$\pi_{i}$ & $U[40,200]$ & $U[40,200]$ & Cota et al. (2018) \\
\hline$V_{l}$ & $1.2,1,0.8$ & $1.2,1.1,1,0.9,0.8$ & $\begin{array}{l}\text { Mansouri et al. (2016), Ahilan et al. } \\
\text { (2013), Cota et al. (2018) }\end{array}$ \\
\hline$\lambda_{l}$ & $1.5,1,0.6$ & $\begin{array}{l}1.5,1.25,1,0.8 \\
0.6\end{array}$ & $\begin{array}{l}\text { Mansouri et al. (2016), Ahilan et al. } \\
\text { (2013), Cota et al. (2018) }\end{array}$ \\
\hline
\end{tabular}

\subsection{Metric description}

The quality of the set of non-dominated solutions found by a method can be analyzed under three aspects: convergence, extension, and distribution. Convergence refers to the proximity of this set to the Paretooptimal front or to the reference set. In turn, the extension assesses the breadth of the region covered by this set of non-dominated solutions. Finally, the distribution refers to the uniformity of the spacing between the solutions within the set.

The hypervolume metric is sensitive for convergence and extension and the HCC metric, in turn, is sensitive for distribution and extension. 


\subsubsection{Hypervolume}

The hypervolume or $\mathrm{S}$ metric is a measure of quality often used to compare results from multi-objective algorithms and it was proposed by Zitzler and Thiele (1998). This metric has the ability to provide a combined estimate of convergence and diversity of a set of solutions (Deb, 2014). The hypervolume of a non-dominated set measures the area covered or dominated by this set's points, limited by a Reference Point $(R P)$. In maximization problems, it is common to use the point $(0 ; 0)$, while in minimization problems, an upper bound, also known as the Nadir point, is used to limit this area. In Figure 7, the shaded area defines the hypervolume of the set of non-dominated solutions $\mathcal{A}$ for a problem with two objective functions, in which the point $\left(\max _{x} ; \max _{y}\right)$ defines the upper limit. We denote by $H V(\mathcal{A})$ the hypervolume of a set of non-dominated solutions $\mathcal{A}$ relative to a reference point (Deb, 2014).

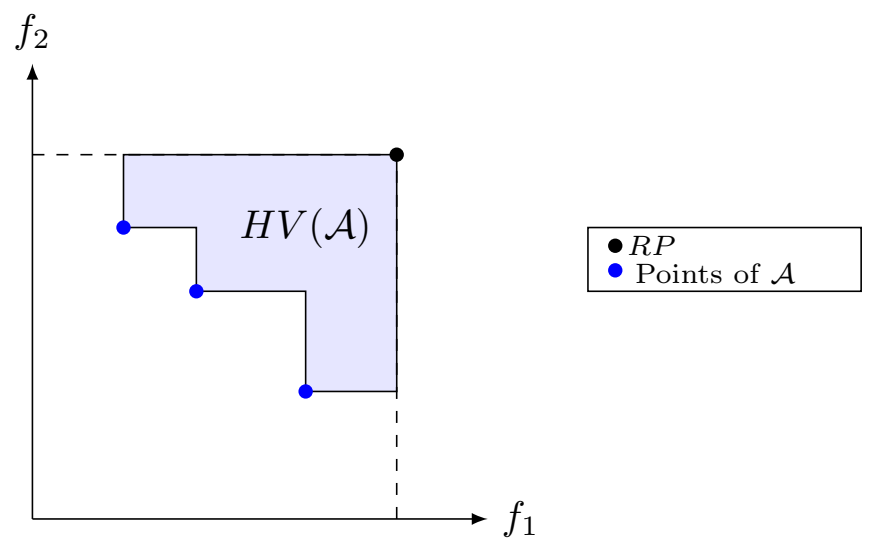

Figure 7. Hypervolume for set $\mathcal{A}$

\subsubsection{HCC}

Hierarchical Cluster Counting (HCC) is a metric proposed by Guimaraes et al. (2009) to evaluate the quality of non-dominated sets that were obtained by multi-objective optimization algorithms. It is based on hierarchical clustering techniques, such as the Sphere Counting (SC) (Wanner et al., 2006) metric. According to Guimaraes et al. (2009), the extent and uniformity of a non-dominated set is directly proportional to the HCC value calculated for it.

We calculate the HCC for a set of points $\mathcal{A}$ as follows:

1. Initially, we create a grouping for each point in the set, and consider that each group created is a sphere of radius equal to zero;

2. Then, we calculate the minimum distances of fusion, which is a new assumed value for the radius of the spheres capable of decreasing the number of clusters;

3. We group the points into the same cluster;

4. We repeat steps 2 and 3 until all the points belong to the same grouping;

5. We obtain the HCC value by adding, in each iteration, the product between the distances of fusion and the amount of grouping formed.

Consider Figure 8, which illustrates the steps to calculate the HCC for a six-point non-dominated set. Figure 8(a) shows the first cluster in which each point is in a different sphere with radius zero. Figure 8(b) shows the points grouped into five spheres, each with radius $r_{1}$. Figure 8(c) shows the points grouped into four spheres, each with radius $r_{2}$. Figure 8(d) shows, in the Cartesian plane, the relationship between the number of clusters and the the radius of each cluster. The gray region area represents the value of the HCC metric for the set shown in Figure 8. 


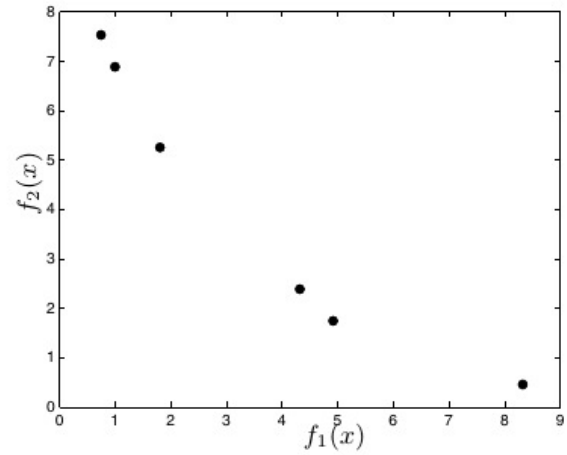

(a) Data

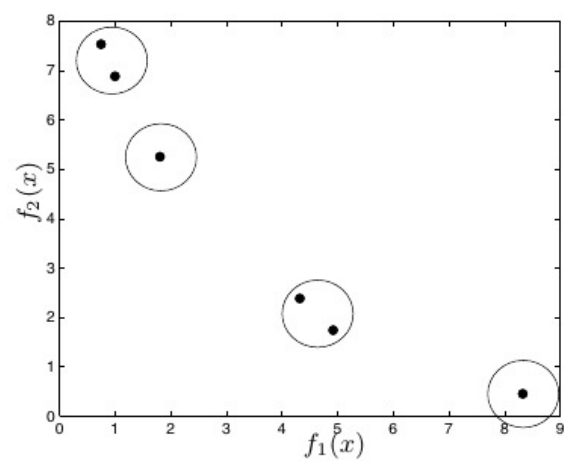

(c) Data grouped into 4 clusters

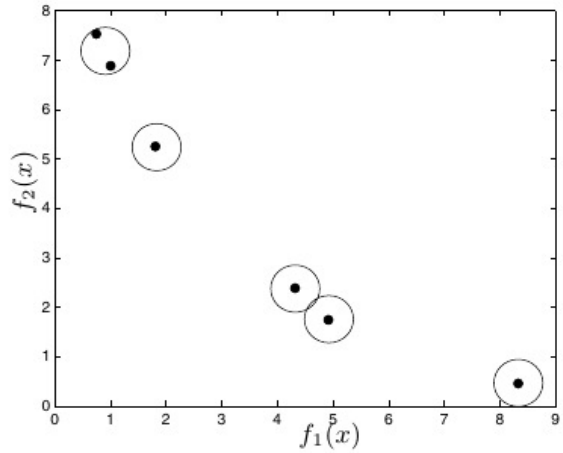

(b) Data grouped into 5 clusters

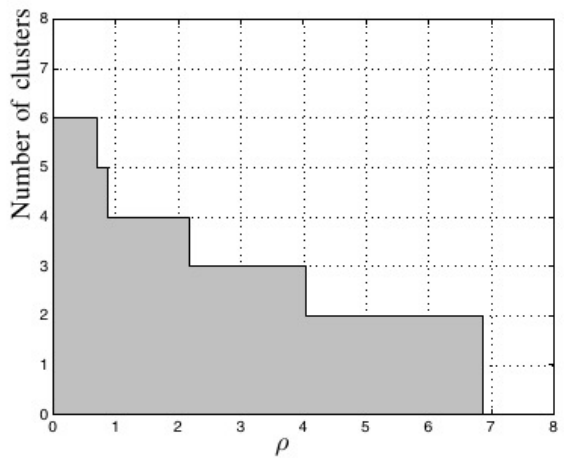

(d) Function $s(\rho)$

Figure 8. Example of how to calculate the HCC metric (Guimaraes et al., 2009)

\subsection{Tuning of algorithms' parameters}

The parameter values used in the NSGA-II, MOGA, and NSGA-I algorithms can affect its performance.

Irace is a software encoded in the $\mathrm{R}$ language that automatically performs an iterative procedure to find the most appropriate optimization algorithm settings.

Table 5 shows the test scenarios used. In the first column, we present the description of each NSGA-II parameter; in the second column, the set of values tested for each parameter, and in the third column, the best value returned by Irace.

Table 5. Test scenarios for algorithms' parameters

\begin{tabular}{lllc}
\hline Method & Description & Tested values & Irace best value \\
\hline \multirow{2}{*}{ NSGA-II } & Population size $\left(\right.$ size $\left._{\text {pop }}\right)$ & $80,90,100,110$ & 110 \\
& Probability of mutation & $0.04,0.05,0.06,0.07$ & 0.05 \\
\hline \multirow{2}{*}{ MOGA } & Population size $\left(\right.$ size $\left._{\text {pop }}\right)$ & $80,90,100,110$ & 80 \\
& Probability of mutation & $0.04,0.05,0.06,0.07$ & 0.06 \\
\hline \multirow{2}{*}{ NSGA-I } & Population size $\left(\right.$ size $\left._{\text {pop }}\right)$ & $80,90,100,110$ & 80 \\
& Probability of mutation & $0.04,0.05,0.06,0.07$ & 0.06 \\
\hline
\end{tabular}

\subsection{Results}

In this section, we presented the results of two experiments used to evaluate the NSGA-II algorithm's performance. First, we compare the NSGA-II results with those of the weighted sum method in instances with up to 10 jobs and 2 machines. Then, we compared the performance of the NSGA-II algorithm with that of the MOGA and NSGA-I algorithms in larger instances, with up to 750 jobs and 20 machines. In both cases, we executed the algorithms 30 times in each instance. 
We used the Relative Percentage Deviation $\left(R P D_{i}^{H V}\right)$ to evaluate the HV metric for each method $A l g$ and instance $i$. It is calculated by Equation (16):

$$
R P D_{i}^{H V}(A l g)=\frac{H V_{i}^{R S}-H V_{i}^{v}}{H V_{i}^{R S}}
$$

where $H V_{i}^{R S}$ is the hypervolume value of the reference set in 30 executions of the algorithm Alg in the instance $i$. $v$ can assume three values: $\min$, max and $a v g$, representing, respectively, the smallest, the largest, and the average of the hypervolume in 30 executions of the algorithm in the instance $i$.

\subsubsection{NSGA-II $\times$ Gurobi}

In this section, we reported the results of the NSGA-II algorithm and the exact method in the set of instances set1.

Table 6 shows the reference set data for these instances. In this table, the first two columns show the instance identifier and name, respectively. The next two columns show the number of jobs and machines, respectively. The fifth column presents the hypervolume of this reference set. Finally, the last column presents the reference point $\left(C_{\max } ; T E C\right)$ used to calculate the hypervolume of each instance.

Table 6. Reference set data in the set1

\begin{tabular}{clccrc}
\hline \# ID & \# Instance & n & m & HV & RP \\
\hline 1 & 6_2_1439_3_S_1-9 & 6 & 2 & $6,406.67$ & $(250 ; 239.91)$ \\
2 & 7_2_1439_3_S_1-9 & 7 & 2 & $15,918.87$ & $(400 ; 260.68)$ \\
3 & 8_2_1439_3_S_1-9 & 8 & 2 & $3,338.29$ & $(260 ; 302.58)$ \\
4 & 9_2_1439_3_S_1-9 & 9 & 2 & $22,256.33$ & $(440 ; 357.69)$ \\
5 & 10_2_1439_3_S_1-9 & 10 & 2 & $31,789.09$ & $(500 ; 370.68)$ \\
\hline
\end{tabular}

Tables 7 and 8 show the method results concerning the $R P D^{H V}$ and the HCC metrics. In these tables, the first column identifies the instance. The next three columns show the minimum, maximum and average values of the $R P D^{H V}$ and HCC, respectively, concerning the NSGA-II method. The fifth column shows the standard deviation of the results. The seventh column shows the upper bound (UB) returned by the exact method concerning the $R P D^{H V}$ and HCC metrics. Finally, the sixth and eighth columns show the times, in seconds, of the NSGA-II and the exact method, respectively.

Table 7. $R P D^{H V}$ and runtime of the methods in the set 1

\begin{tabular}{c|rrrrr|rr}
\hline \multirow{2}{*}{ \# ID } & \multicolumn{5}{|c|}{ NSGA-II } & \multicolumn{2}{c}{ Gurobi } \\
\cline { 2 - 8 } & $\min$ & $\max$ & avg & sd & time (s) & UB & time (s) \\
& $(\%)$ & $(\%)$ & $(\%)$ & & & & \\
\hline 1 & 0.00 & 0.00 & 0.00 & 0.00 & 4.16 & 1.89 & 172.09 \\
2 & 0.68 & 0.68 & 0.68 & 0.00 & 4.85 & 1.12 & 549.86 \\
3 & 0.00 & 0.00 & 0.00 & 0.00 & 5.54 & 0.34 & $2,140.40$ \\
4 & 0.00 & 0.75 & 0.62 & 0.28 & 6.24 & 5.12 & $8,312.92$ \\
5 & 0.66 & 0.89 & 0.66 & 0.04 & 6.93 & 1.51 & $39,396.63$ \\
\hline
\end{tabular}

We can see in Table 6 that in the set of instances set1, the $R P D^{H V}$ of the NSGA-II algorithm is lower in all comparisons (min, max e avg) compared to the exact method. We can also verify that the standard deviation of the NSGA-II algorithm in instances with up to 8 jobs (ID 1,2,3) is equal to zero. In other words, in these instances, all NSGA-II executions obtained the same set non-dominated. We also can note that the execution time of the NSGA-II is much less than that of the exact algorithm.

Concerning Table 8, we noted that the NSGA-II algorithm has a higher HCC value than the exact method in all comparisons. Thus, we can conclude that the non-dominated set of the NSGA-II method has better diversity and uniformity.

Figure 9 presents the non-dominated sets obtained by an NSGA-II execution and the other using the exact method in two randomly selected instances. The first instance has 6 jobs and 2 machines, and the 
Table 8. HCC and runtime of the methods in the set1

\begin{tabular}{c|rrrrl|rr}
\hline \multirow{2}{*}{ \# ID } & \multicolumn{5}{|c|}{ NSGA-II } & \multicolumn{2}{c}{ Gurobi } \\
\cline { 2 - 8 } & $\min$ & $\max$ & avg & sd & time $(\mathrm{s})$ & UB & time (s) \\
\hline 1 & 305.16 & 305.16 & 305.16 & 0.00 & 4.16 & 253.78 & 172.09 \\
2 & 75.87 & 75.87 & 75.87 & 0.00 & 4.85 & 43.52 & 549.86 \\
3 & 356.33 & 356.33 & 356.33 & 0.00 & 5.54 & 215.71 & $2,140.40$ \\
4 & 70.63 & 80.64 & 78.97 & 12.80 & 6.24 & 6.56 & $8,312.92$ \\
5 & 84.98 & 87.79 & 87.70 & 2.05 & 6.93 & 77.15 & $39,393.63$ \\
\hline
\end{tabular}

second has 10 jobs and 2 machines. In this figure, the blue dots represent the solutions of the NSGA-II, and the red dots represent the solutions of the exact method. The $x$ axis represents the makespan, and the $y$ axis represents the total energy cost.

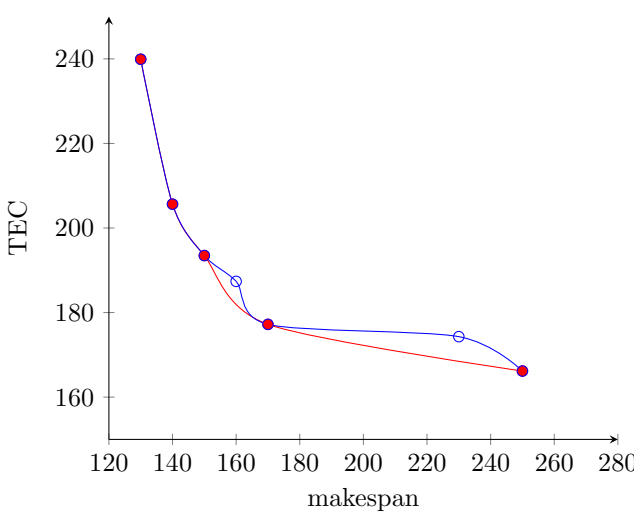

(a) Instance with 6 jobs and 2 machines

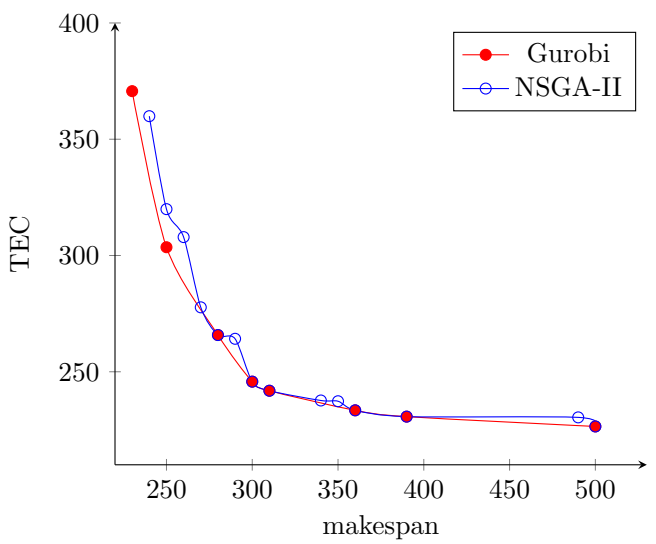

(b) Instance with 10 jobs and 2 machines

Figure 9. Frontiers found by NSGA-II and Gurobi methods

We can notice in Figure 9(a) that the NSGA-II non-dominated set contains all the solutions found by the exact method, plus two additional solutions. In this example, the two methods have the same hand, Figure 9(b) shows that the non-dominated set contains six of the eight solutions found by the exact method and eight other solutions. In this example, the exact method showed better amplitude than the NSGA-II, but this obtained higher cardinality than the exact method.

Considering these results, we observed that the NSGA-II finds good quality solutions and requires less computational time than the exact method.

\subsubsection{NSGA-II in large instances compared with other literature algorithms}

Here, we presented the results of the NSGA-II, MOGA, and NSGA-I algorithms in the set of instances set2.

Table 9 shows the reference set data for the instances of set2. Its organization follows the same description as the previous section's tables.

Tables 10 and 11 report the $R P D^{H V}$ and $\mathrm{HCC}$ values, respectively, of the algorithms in the set of instances set2. As can be seen, NSGA-II achieved the best average results regarding hypervolume in all instances. On the other hand, it won in $2 / 3$ of the instances concerning the HCC. These results indicate that the NSGA-II algorithm outperforms MOGA and NSGA-I algorithms concerning these metrics. 
Table 9. Reference set data in the set2

\begin{tabular}{clccrl}
\hline \# ID & \# Instance & $\mathrm{n}$ & $\mathrm{m}$ & $\mathrm{HV}$ & $\mathrm{RP}$ \\
\hline 1 & 50_10_1439_5_S_1-9 & 50 & 10 & 56,872 & $(280 ; 452.653)$ \\
2 & 50_10_1439_5_S_1-124 & 50 & 10 & 181,673 & $(456 ; 909.559)$ \\
3 & 50_20_1439_5_S_1-9 & 50 & 20 & 15,933 & $(114 ; 323.339)$ \\
4 & 50_20_1439_5_S_1-124 & 50 & 20 & 151,884 & $(392 ; 642.568)$ \\
5 & 250_10_1439_5_S_1-9 & 250 & 10 & $1,488,058$ & $(1457 ; 2245.16)$ \\
6 & 250_10_1439_5_S_1-124 & 250 & 10 & $6,986,225$ & $(4374 ; 2930.02)$ \\
7 & 250_20_1439_5_S_1-9 & 250 & 20 & 526,773 & $(420 ; 2570.86)$ \\
8 & 250_20_1439_5_S_1-124 & 250 & 20 & $1,383,849$ & $(988 ; 3179.8)$ \\
9 & 750_10_1439_5_S_1-9 & 750 & 10 & $7,678,168$ & $(3665 ; 5630.8)$ \\
10 & 750_10_1439_5_S_1-124 & 750 & 10 & $105,897,123$ & $(19400 ; 9442.33)$ \\
11 & 750_20_1439_5_S_1-9 & 750 & 20 & $3,791,005$ & $(1364 ; 5573.7)$ \\
12 & 750_20_1439_5_S_1-124 & 750 & 20 & $34,971,819$ & $(7740 ; 7065.71)$ \\
\hline
\end{tabular}

Table 10. $R P D^{H V}$ and runtime of the algorithms in the set2. The best average values are highlighted in bold.

\begin{tabular}{c|cccc|cccc|cccc|r}
\hline \multirow{4}{*}{ \# ID } & \multicolumn{4}{|c|}{ MOGA } & \multicolumn{4}{|c|}{ NSGA-I } & \multicolumn{4}{c|}{ NSGA-II } & \\
\cline { 2 - 7 } & $\min$ & $\max$ & $a v g$ & $s d$ & $\min$ & $\max$ & $a v g$ & $s d$ & $\min$ & $\max$ & $a v g$ & $s d$ & \multirow{2}{*}{ time (s) } \\
& $(\%)$ & $(\%)$ & $(\%)$ & & $(\%)$ & $(\%)$ & $(\%)$ & & $(\%)$ & $(\%)$ & $(\%)$ & & \\
\hline 1 & 0.08 & 0.28 & 0.19 & 0.06 & 0.04 & 0.18 & $\mathbf{0 . 1 0}$ & 0.03 & 0.01 & 0.18 & $\mathbf{0 . 1 0}$ & 0.03 & 115.13 \\
2 & 0.03 & 0.20 & 0.12 & 0.04 & 0.05 & 0.20 & 0.11 & 0.04 & 0.03 & 0.20 & $\mathbf{0 . 1 0}$ & 0.04 & 115.13 \\
3 & 0.04 & 0.27 & 0.15 & 0.06 & 0.07 & 0.25 & 0.15 & 0.05 & 0.05 & 0.21 & $\mathbf{0 . 1 2}$ & 0.05 & 149.79 \\
4 & 0.04 & 0.16 & 0.11 & 0.03 & 0.02 & 0.16 & 0.10 & 0.03 & 0.02 & 0.17 & $\mathbf{0 . 0 9}$ & 0.03 & 149.79 \\
5 & 0.22 & 0.27 & 0.24 & 0.02 & 0.02 & 0.09 & 0.06 & 0.02 & 0.01 & 0.04 & $\mathbf{0 . 0 3}$ & 0.01 & 575.65 \\
6 & 0.01 & 0.11 & 0.06 & 0.03 & 0.01 & 0.04 & $\mathbf{0 . 0 2}$ & 0.01 & 0.01 & 0.03 & $\mathbf{0 . 0 2}$ & 0.01 & 575.65 \\
7 & 0.13 & 0.36 & 0.24 & 0.06 & 0.05 & 0.14 & 0.10 & 0.02 & 0.01 & 0.08 & $\mathbf{0 . 0 5}$ & 0.02 & 748.93 \\
8 & 0.03 & 0.11 & $\mathbf{0 . 0 7}$ & 0.02 & 0.04 & 0.12 & 0.08 & 0.02 & 0.04 & 0.12 & $\mathbf{0 . 0 7}$ & 0.02 & 748.93 \\
9 & 0.21 & 0.33 & 0.24 & 0.03 & 0.02 & 0.06 & 0.04 & 0.01 & 0.01 & 0.02 & $\mathbf{0 . 0 1}$ & 0.00 & $1,726.94$ \\
10 & 0.10 & 0.15 & 0.13 & 0.01 & 0.01 & 0.03 & 0.02 & 0.00 & 0.01 & 0.02 & $\mathbf{0 . 0 1}$ & 0.00 & $1,727.94$ \\
11 & 0.13 & 0.21 & 0.17 & 0.02 & 0.05 & 0.17 & 0.10 & 0.03 & 0.01 & 0.05 & $\mathbf{0 . 0 2}$ & 0.01 & $2,246.80$ \\
12 & 0.04 & 0.07 & 0.06 & 0.01 & 0.01 & 0.02 & 0.02 & 0.00 & 0.00 & 0.02 & $\mathbf{0 . 0 1}$ & 0.00 & $2,246.80$ \\
\hline
\end{tabular}




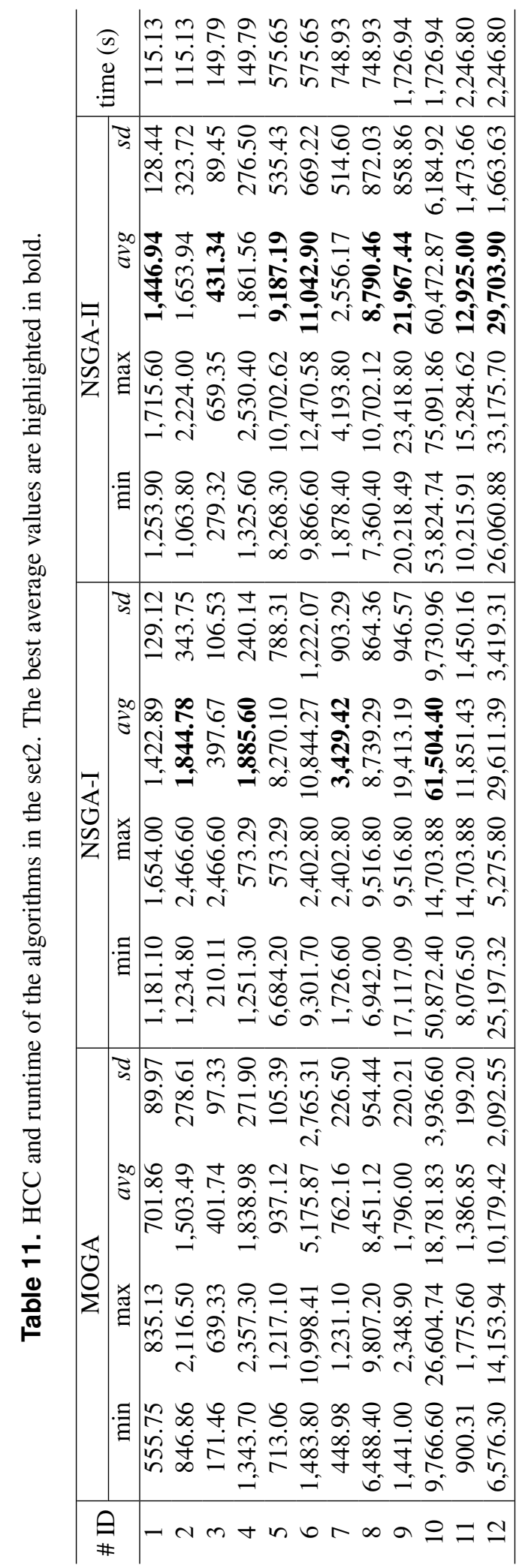


Figures 10(a)-(b) illustrate the Pareto front obtained from each algorithm in two different instances. The first instance has 50 jobs and 20 machines, and the second has 750 jobs and 10 machines. As can be seen, the NSGA-II produced sets of non-dominated solutions with good convergence, diversity, uniformity, and amplitude when compared with others algorithms.

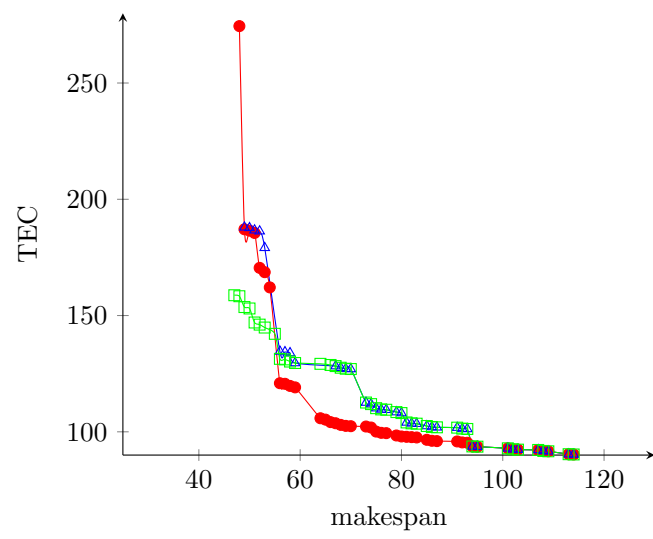

(a) Instance with 50 jobs and 20 machines

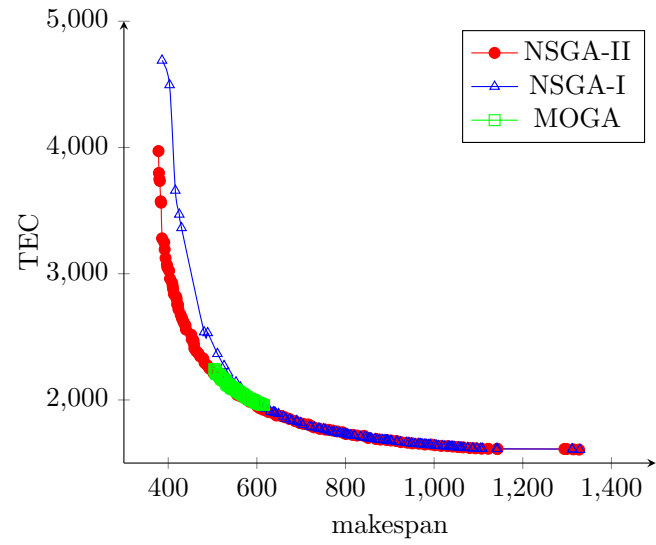

(b) Instance with 750 jobs and 10 machines

Figure 10. The Pareto front obtained from each algorithm

Figures 11 and 12 show the boxplot of the RPD and HCC results, respectively.

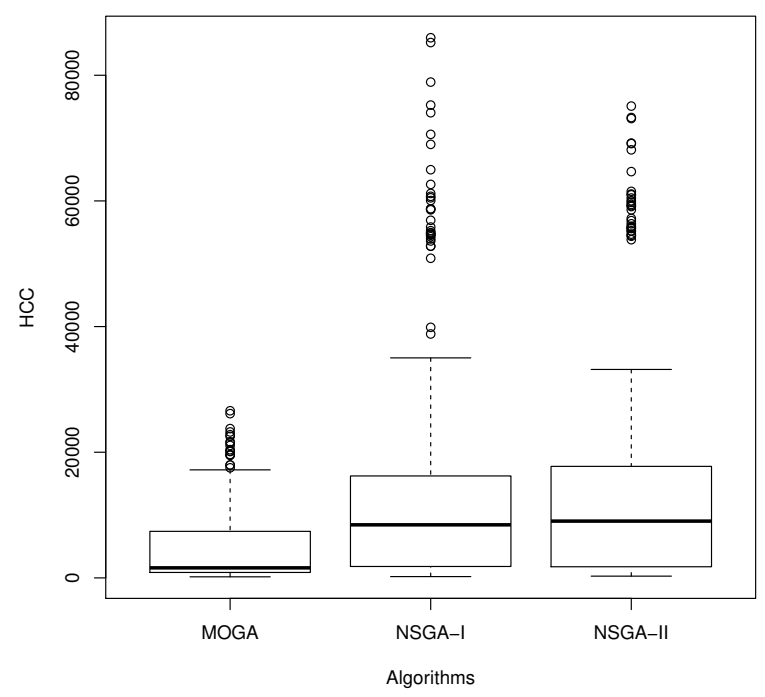

Figure 11. Boxplot of the $\mathrm{RPD}^{H V}$ results

To verify if the differences between the results presented by the algorithms are statistically significant, we performed the hypothesis tests below.

$$
\left\{\begin{array}{l}
H_{0}: \mu_{1}=\mu_{2}=\mu_{3} \\
H_{1}: \exists i, j \mid \mu_{i} \neq \mu_{j}
\end{array}\right.
$$

In the first test, $\mu_{1}, \mu_{2}$, and $\mu_{3}$ represent the average RPD ${ }^{H V}$ for NSGA-II, MOGA, and NSGA-I, respectively. In the second test, $\mu_{1}, \mu_{2}$, and $\mu_{3}$ represent the average HCC for the algorithms in the same sequence. 


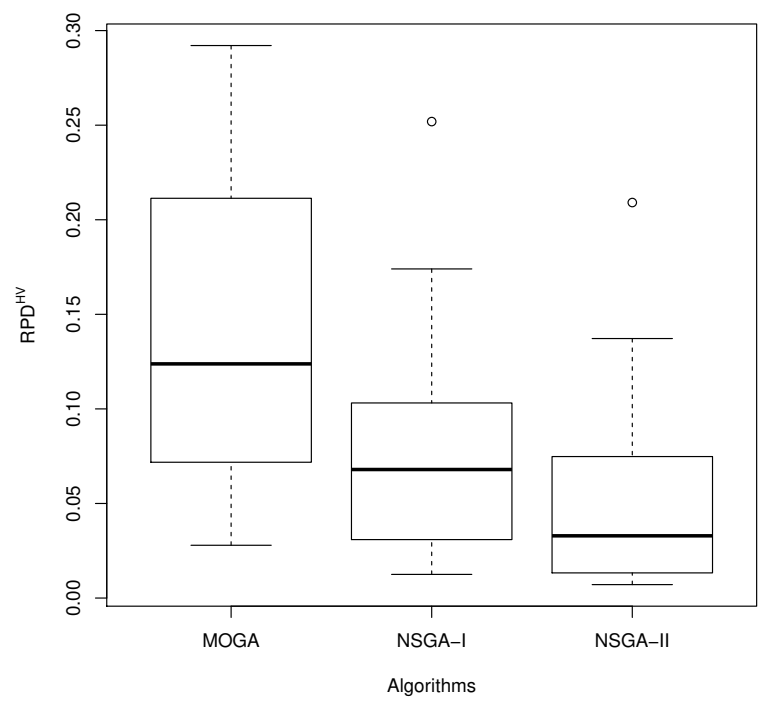

Figure 12. Boxplot of the $\mathrm{HCC}$ results

Before performing the hypothesis tests, we need to choose the test type, parametric or non-parametric. Generally, parametric tests are more powerful; however, to use them, it is necessary to satisfy three assumptions:

1. Normality: every sample must originate from a population with normal distribution,

2. Independence: the samples must be independent of each other,

3. Homoscedasticity: there must be equality of variances across samples.

We applied the Shapiro-Wilk normality test to the samples with the $\mathrm{RPD}^{H V}$ and HCC values from each algorithm and showed its results in Table 12.

Table 12. $p$-values of the Shapiro-Wilk normality test concerning $\mathrm{RPD}^{H V}$ and $\mathrm{HCC}$ values

\begin{tabular}{lcc}
\hline Algorithm & RPD $^{H V}$ & HCC \\
\hline MOGA & 0.0072 & $2.2 \mathrm{e}-16$ \\
NSGA-I & 0.0002 & $2.2 \mathrm{e}-16$ \\
NSGA-II & $2.289 \mathrm{e}-06$ & $2.2 \mathrm{e}-16$ \\
\hline
\end{tabular}

With confidence level of $95 \%(\alpha=0.05)$, we can say that the results presented in Table 12 not present evidence that the results of the algorithms come from a population with normal distribution.

Thus, we applied the Paired Wilcoxon signed-rank non-parametric test (Wilcoxon, 1945). Table 13 reports the results of this test obtained by the NSGA-II, MOGA, and NSGA-I algorithms for the samples of the $\mathrm{RPD}^{H V}$ and $\mathrm{HCC}$ values.

Table 13. $p$-values of the paired Wilcoxon signed-rank test concerning $\operatorname{RPD}^{H V}$ and $\mathrm{HCC}$ values $(\alpha=$ $0.05)$.

\begin{tabular}{lcc}
\hline Comparison & RPD $^{H V}$ & HCC \\
\hline MOGA $v s$ NSGA-I & $8.0 \mathrm{e}-08$ & $2 \mathrm{e}-16$ \\
MOGA $v$ NSGA-II & $7.8 \mathrm{e}-10$ & $2 \mathrm{e}-16$ \\
NSGA-I $v s$ NSGA-II & $1.8 \mathrm{e}-08$ & 0.0001 \\
\hline
\end{tabular}


According to Table 13, there are significant statistical difference between each pair of algorithms. Thus, these tests confirm the results in Tables 10 and 11, indicating that NSGA-II outperforms both MOGA and NSGA-I.

\section{CONCLUSIONS}

This paper addressed the unrelated parallel machine scheduling problem with sequence-dependent setup times for minimizing the total energy cost and the makespan.

To solve it, we developed a mixed-integer linear programming formulation and applied the weighted sum method to generate sets of non-dominated solutions to the problem. Considering that this formulation could not solve larger instances of the problem, we adapted the NSGA-II algorithm to deal with them.

To test the two solution methods, we adapted instances of the literature to contemplate all the problem's characteristics. We divided these instances into two groups. The first group consists of small instances with up to 10 jobs and 2 machines, while the second group contains large instances, with up to 750 jobs and 20 machines. We evaluated the methods concerning the hypervolume and HCC metrics.

Initially, we used part of the set of instances to tuning the parameter values of the NSGA-II algorithm. To this end, we used the Irace package.

We validated the NSGA-II algorithm in small instances, comparing its results with those produced by the exact method. The NSGA-II algorithm showed good convergence and diversity. Besides, it spent much shorter CPU time than that required by the exact method.

In large instances, the results showed that the NSGA-II outperforms, with $95 \%$ confidence level, MOGA and NSGA-I algorithms concerning the hypervolume and HCC metrics. Thus, the proposed algorithm finds non-dominated solutions with good convergence, diversity, uniformity, and amplitude.

As future work, we suggest testing other crossover and mutation operators for the NSGA-II. Besides, we intend to implement other multi-objective algorithms, such as Strength Pareto Evolutionary Algorithm 2 (SPEA2), Niched Pareto Genetic Algorithm (NPGA), Pareto Envelope-based Selection Algorithm II (PESA-II), Multi-objective Variable Neighborhood Search (MOVNS), and Multi-objective Evolutionary Algorithm Based on Decomposition (MOEA/D).

\section{ACKNOWLEDGMENTS}

The authors thank the Universidade Federal de Ouro Preto, the Instituto Tecnologico Vale, and the Universidade Federal dos Vales do Jequitinhonha e Mucuri for partially supporting the development of this research.

\section{REFERENCES}

Ahilan, C., Kumanan, S., Sivakumaran, N., and Dhas, J. E. R. (2013). Modeling and prediction of machining quality in cnc turning process using intelligent hybrid decision making tools. Applied Soft Computing, 13(3):1543-1551.

Babazadeh, H., Alavidoost, M., Zarandi, M. F., and Sayyari, S. (2018). An enhanced nsga-ii algorithm for fuzzy bi-objective assembly line balancing problems. Computers \& industrial engineering, 123:189208.

BEIS (2020). Industrial electricity prices in the IEA 2020; U.K. Department for Business Energy Industrial Strategy. Available at https://assets.publishing.service.gov. uk/government/uploads/system/uploads/attachment_data/file/895162/ table_531.xlsx, accessed on February 28, 2020.

Che, A., Zhang, S., and Wu, X. (2017). Energy-conscious unrelated parallel machine scheduling under time-of-use electricity tariffs. Journal of Cleaner Production, 156:688-697.

Cheng, J., Chu, F., and Zhou, M. (2018). An improved model for parallel machine scheduling under timeof-use electricity price. IEEE Transactions on Automation Science and Engineering, 15(2):896-899.

Cheng, J., Wu, P., and Chu, F. (2019). Mixed-integer programming for unrelated parallel machines scheduling problem considering electricity cost and makespan penalty cost. In 2019 International

500 Conference on Industrial Engineering and Systems Management (IESM), pages 1-5, Shanghai, China. IEEE. 
Cota, L. P., Coelho, V. N., Guimarães, F. G., and Souza, M. J. F. (2018). Bi-criteria formulation for green scheduling with unrelated parallel machines with sequence-dependent setup times. International Transactions in Operational Research, 28:996-1017.

Cota, L. P., Guimarães, F. G., Ribeiro, R. G., Meneghini, I. R., de Oliveira, F. B., Souza, M. J. F., and Siarry, P. (2019). An adaptive multi-objective algorithm based on decomposition and large neighborhood search for a green machine scheduling problem. Swarm and Evolutionary Computation, 51:100601.

Deb, K. (2014). Multi-objective optimization. In Search methodologies, pages 403-449. Springer.

Deb, K., Karthik, S., et al. (2007). Dynamic multi-objective optimization and decision-making using modified nsga-ii: a case study on hydro-thermal power scheduling. In International conference on evolutionary multi-criterion optimization, pages 803-817. Springer.

Deb, K., Pratap, A., Agarwal, S., and Meyarivan, T. (2002). A fast and elitist multiobjective genetic algorithm: NSGA-II. IEEE transactions on evolutionary computation, 6(2):182-197.

Ding, J., Song, S., Zhang, R., Chiong, R., and Wu, C. (2016). Parallel machine scheduling under time-ofuse electricity prices: New models and optimization approaches. IEEE Transactions on Automation Science and Engineering, 13(2):1138-1154.

Ebrahimi, A., Jeon, H. W., Lee, S., and Wang, C. (2020). Minimizing total energy cost and tardiness penalty for a scheduling-layout problem in a flexible job shop system: A comparison of four metaheuristic algorithms. Computers \& Industrial Engineering, 141:106295.

EIA (2016). International energy outlook 2016 with projections to 2040. Technical Report DOE/EIA0484, U.S. Energy Information Administration, U.S. Department of Energy, Washington. Available at https://www.eia.gov/outlooks/ieo/pdf/0484 (2016).pdf, accessed on February 1, 2020.

Feo, T. A. and Resende, M. G. (1995). Greedy randomized adaptive search procedures. Journal of global optimization, 6(2):109-133.

Garey, M. R. and Johnson, D. S. (1979). Computers and intractability. A guide to the theory of NPcompleteness. freeman San Francisco.

Guimaraes, F. G., Wanner, E. F., and Takahashi, R. H. C. (2009). A quality metric for multi-objective optimization based on hierarchical clustering techniques. In 2009 IEEE Congress on Evolutionary Computation, pages 3292-3299.

Gurobi Optimization, L. (2020). Gurobi optimizer reference manual. Available at http://wWw . gurobi. com.

Kopanos, G. M., Laínez, J. M., and Puigjaner, L. (2009). An efficient mixed-integer linear programming scheduling framework for addressing sequence-dependent setup issues in batch plants. Industrial \& Engineering Chemistry Research, 48(13):6346-6357.

Kurniawan, B., Gozali, A., Weng, W., and Fujimura, S. (2017). A genetic algorithm for unrelated parallel machine scheduling minimizing makespan cost and electricity cost under time-of-use (tou) tariffs with job delay mechanism. In 2017 IEEE International Conference on Industrial Engineering and Engineering Management (IEEM), pages 583-587. IEEE.

Liang, P., Yang, H., Liu, G.-s., and Guo, J. (2015). An ant optimization model for unrelated parallel machine scheduling with energy consumption and total tardiness. Mathematical Problems in Engineering, 2015.

Liu, Y., Dong, H., Lohse, N., Petrovic, S., and Gindy, N. (2014). An investigation into minimising total energy consumption and total weighted tardiness in job shops. Journal of Cleaner Production, 65:87-96.

López-Ibáñez, M., Dubois-Lacoste, J., Cáceres, L. P., Birattari, M., and Stützle, T. (2016). The irace package: Iterated racing for automatic algorithm configuration. Operations Research Perspectives, 3:43-58.

Mansouri, S. A., Aktas, E., and Besikci, U. (2016). Green scheduling of a two-machine flowshop: Trade-off between makespan and energy consumption. European Journal of Operational Research, 248(3):772-788.

Marler, R. T. and Arora, J. S. (2004). Survey of multi-objective optimization methods for engineering. Structural and multidisciplinary optimization, 26(6):369-395.

Murata, T., Ishibuchi, H., et al. (1995). Moga: multi-objective genetic algorithms. In IEEE international conference on evolutionary computation, volume 1, pages 289-294.

Pinedo, M. L. (2016). Scheduling: Theory, Algorithms, and Systems. Springer International Publishing. 
Rego, M. F., Cota, L. P., and Souza, M. J. F. (2021). Instances for the upmsp with sequence-dependent setup times under time-of-use electricity price. Available at https://github.com/marcelofr/ UPMSP_ME_INSTANCE.

Ropke, S. and Pisinger, D. (2006). An adaptive large neighborhood search heuristic for the pickup and delivery problem with time windows. Transportation science, 40(4):455-472.

Rosa, B. F. and Souza, M. J. F. (2009). Uma nova formulaçao de programaçao matemática indexada no tempo para uma classe de problemas de sequenciamento em uma máquina. Anais do XLI Simpósio Brasileiro de Pesquisa Operacional, pages 2898-2909.

Saberi-Aliabad, H., Reisi-Nafchi, M., and Moslehi, G. (2020). Energy-efficient scheduling in an unrelated parallel-machine environment under time-of-use electricity tariffs. Journal of Cleaner Production, 249:119393.

Shrouf, F., Ordieres-Meré, J., García-Sánchez, A., and Ortega-Mier, M. (2014). Optimizing the production scheduling of a single machine to minimize total energy consumption costs. Journal of Cleaner Production, 67:197-207.

Srinivas, N. and Deb, K. (1994). Muiltiobjective optimization using nondominated sorting in genetic algorithms. Evolutionary computation, 2(3):221-248.

Tsao, Y., Thanh, V., and Hwang, F. (2020). Energy-efficient single-machine scheduling problem with controllable job processing times under differential electricity pricing. Resources, Conservation and Recycling, 161:104902.

Vallada, E. and Ruiz, R. (2011). A genetic algorithm for the unrelated parallel machine scheduling problem with sequence dependent setup times. European Journal of Operational Research, 211(3):612-622.

Wang, H., Fu, Y., Huang, M., Huang, G. Q., and Wang, J. (2017). A nsga-ii based memetic algorithm for multiobjective parallel flowshop scheduling problem. Computers \& Industrial Engineering, 113:185194.

Wang, S., Liu, M., Chu, F., and Chu, C. (2016). Bi-objective optimization of a single machine batch scheduling problem with energy cost consideration. Journal of Cleaner Production, 137:1205-1215.

Wanner, E. F., Guimaraes, F. G., Takahashi, R. H., and Fleming, P. J. (2006). A quadratic approximationbased local search procedure for multiobjective genetic algorithms. In 2006 IEEE International Conference on Evolutionary Computation, pages 938-945. IEEE.

Wilcoxon, F. (1945). Some uses of statistics in plant pathology. Biometrics Bulletin, 1(4):41-45.

Willeke, S., Ullmann, G., and Nyhuis, P. (2016). Method for an energy-cost-oriented manufacturing control to reduce energy costs: Energy cost reduction by using a new sequencing method. In 2016 International Conference on Industrial Engineering, Management Science and Application (ICIMSA), pages 1-5. IEEE.

Wu, X. and Che, A. (2019). A memetic differential evolution algorithm for energy-efficient parallel machine scheduling. Omega, 82:155-165.

Zeng, Y., Che, A., and $\mathrm{Wu}, \mathrm{X}$. (2018). Bi-objective scheduling on uniform parallel machines considering electricity cost. Engineering Optimization, 50(1):19-36.

Zhang, H., Zhao, F., Fang, K., and Sutherland, J. W. (2014). Energy-conscious flow shop scheduling under time-of-use electricity tariffs. CIRP Annals, 63(1):37 - 40.

Zhang, Q. and Li, H. (2007). Moea/d: A multiobjective evolutionary algorithm based on decomposition. IEEE Transactions on evolutionary computation, 11(6):712-731.

Zitzler, E. and Thiele, L. (1998). Multiobjective optimization using evolutionary algorithms - a comparative case study. In Parallel problem solving from nature-PPSN V, pages 292-301. Springer. 